\title{
The KP approximation under a weak Coriolis forcing
}

\author{
Benjamin MELINAND*
}

October 2017

\begin{abstract}
In this paper, we study the asymptotic behavior of weakly transverse water-waves under a weak Coriolis forcing in the long wave regime. We derive the BoussinesqCoriolis equations in this setting and we provide a rigorous justification of this model. Then, from these equations, we derive two other asymptotic models. When the Coriolis forcing is weak, we fully justify the rotation-modified Kadomtsev-Petviashvili equation (also called Grimshaw-Melville equation). When the Coriolis forcing is very weak, we rigorously justify the Kadomtsev-Petviashvili equation. This work provides the first mathematical justification of the KP approximation under a Coriolis forcing.
\end{abstract}

\section{Introduction}

We consider the motion of an inviscid, incompressible fluid under the influence of the gravity $\boldsymbol{g}=-g \boldsymbol{e}_{\boldsymbol{z}}$ and the rotation of the Earth with a rotation vector $\mathbf{f}=\frac{f}{2} \boldsymbol{e}_{\boldsymbol{z}}$. We assume that the fluid has a constant density $\rho$ and that no surface tension is involved. We assume that the surface is a graph above the still water level and that the seabed is flat. We denote by $X=(x, y) \in \mathbb{R}^{2}$ the horizontal variable and by $z \in \mathbb{R}$ the vertical variable. The fluid occupies the domain $\Omega_{t}:=\left\{(X, z) \in \mathbb{R}^{3},-H<z<\zeta(t, X)\right\}$. We denote by $\mathbf{U}=(\mathbf{V}, \mathrm{w})^{t}$ the velocity in the fluid. Notice that $\mathbf{V}$ is the horizontal component of $\mathbf{U}$ and $\mathrm{w}$ its vertical component. Finally, we assume that the pressure $\mathcal{P}$ is constant at the surface of the fluid. The equations governing such a fluid are the free surface Euler-Coriolis equations ${ }^{(1)}$

$$
\left\{\begin{array}{l}
\partial_{t} \mathbf{U}+\left(\mathbf{U} \cdot \nabla_{X, z}\right) \mathbf{U}+\mathbf{f} \times \mathbf{U}=-\frac{1}{\rho} \nabla_{X, z} \mathcal{P}-g \boldsymbol{e}_{\boldsymbol{z}} \text { in } \Omega_{t}, \\
\operatorname{div} \mathbf{U}=0 \text { in } \Omega_{t},
\end{array}\right.
$$

with the boundary conditions

\footnotetext{
${ }^{*}$ Indiana University. Email : bmelinan@indiana.edu

${ }^{1}$ The centrifugal potential is assumed to be constant and included in the pressure term.
} 


$$
\left\{\begin{array}{l}
\mathcal{P}_{\mid z=\zeta}=P_{0}, \\
\partial_{t} \zeta-\underline{\mathbf{U}} \cdot \mathbf{N}=0, \\
\mathrm{w}_{b}=0
\end{array}\right.
$$

where $P_{0}$ is constant, $\mathbf{N}=\left(\begin{array}{c}-\nabla \zeta \\ 1\end{array}\right), \underline{\mathbf{U}}=\left(\begin{array}{l}\underline{\mathbf{V}} \\ \underline{\mathbf{w}}\end{array}\right)=\mathbf{U}_{\mid z=\zeta}$ and $\mathbf{U}_{b}=\left(\begin{array}{c}\mathbf{V}_{b} \\ \mathbf{w}_{b}\end{array}\right)=\mathbf{U}_{\mid z=-H}$.

In this work, we do not directly work on the free surface Euler-Coriolis equations. We rather consider another formulation called the Castro-Lannes formulation (see [4]). This formulation generalizes the well-known Zakharov/Craig-Sulem formulation $([22,6])$ to a fluid with a rotational component. In [4], Castro and Lannes shown that we can express the free surface Euler equations thanks to the unknowns $\left(\zeta, \mathbf{U}_{/ /}, \boldsymbol{\omega}\right)^{(2)}$ where $\boldsymbol{\omega}=\operatorname{Curl} \mathbf{U}$ is the vorticity of the fluid and

$$
\mathbf{U}_{/ /}=\underline{\mathbf{V}}+\underline{\mathrm{w}} \nabla \zeta .
$$

Then, they provide a system of three equations on these unknowns. In [15], a similar work has been done to take into account the Coriolis forcing. It leads to the following system, called the Castro-Lannes system or the water waves equations with vorticity,

$$
\left\{\begin{array}{l}
\partial_{t} \zeta-\underline{\mathbf{U}} \cdot \mathbf{N}=0 \\
\partial_{t} \mathbf{U}_{/ /}+\nabla \zeta+\frac{1}{2} \nabla\left|\mathbf{U}_{/ /}\right|^{2}-\frac{1}{2} \nabla\left[\left(1+|\nabla \zeta|^{2}\right) \underline{\underline{w}}^{2}\right]+\left(\nabla^{\perp} \cdot \mathbf{U}_{/ /}\right) \underline{\mathbf{V}}^{\perp}+f \underline{\mathbf{V}}^{\perp}=0 \\
\partial_{t} \boldsymbol{\omega}+\left(\mathbf{U} \cdot \nabla_{X, z}\right) \boldsymbol{\omega}=\left(\boldsymbol{\omega} \cdot \nabla_{X, z}\right) \mathbf{U}+f \partial_{z} \mathbf{U}
\end{array}\right.
$$

where $\mathbf{U}=\left(\begin{array}{c}\mathbf{V} \\ \mathrm{w}\end{array}\right)=\mathbf{U}[\zeta]\left(\mathbf{U}_{/ /}, \boldsymbol{\omega}\right)$ is the unique solution in $H^{1}\left(\Omega_{t}\right)$

of the following Div-Curl equation

$$
\left\{\begin{array}{l}
\operatorname{curl} \mathbf{U}=\omega \text { in } \Omega_{t} \\
\operatorname{div} \mathbf{U}=0 \text { in } \Omega_{t} \\
(\underline{\mathbf{V}}+\underline{\mathrm{w}} \nabla \zeta)_{\mid z=\zeta}=\mathbf{U}_{/ /} \\
\mathrm{w}_{b}=0
\end{array}\right.
$$

The main goal of this paper is to study weakly transverse long waves. Therefore, we consider a nondimensionalization of the previous equations. Five physical parameters are involved in this work : the typical amplitude of the surface $a$, the typical longitudinal scale $L_{x}$, the typical transverse scale $L_{y}$, the characteristic water depth $H$ and the typical Coriolis frequency $f$. We introduce four dimensionless parameters

$$
\mu=\frac{H^{2}}{L_{x}^{2}}, \varepsilon=\frac{a}{H}, \text { Ro }=\frac{a \sqrt{g H}}{H f L_{x}} \text { and } \gamma=\frac{L_{x}}{L_{y}} .
$$

\footnotetext{
${ }^{2}$ Notice that Castro and Lannes used the unknowns $\left(\zeta, \frac{\nabla}{\Delta} \cdot \mathbf{U}_{/ /}, \boldsymbol{\omega}\right)$. However, as noticed in [16], the unknowns $\left(\zeta, \mathbf{U}_{/ /}, \boldsymbol{\omega}\right)$ are better to derive shallow water asymptotic models.
} 
The parameter $\mu$ is called the shallowness parameter. The parameter $\varepsilon$ is called the nonlinearity parameter. The parameter Ro is the Rossby number and finally the parameter $\gamma$ is called the transversality parameter. Then, we can nondimensionalize the Euler equations (11) and the Castro-Lannes equations (2) (see Part 1.2). In this work, we study the following asymptotic regime

$$
\mathcal{A}_{\text {boussi }}=\left\{(\mu, \varepsilon, \gamma, \text { Ro }), 0 \leq \mu \leq \mu_{0}, \varepsilon=\mathcal{O}(\mu), \gamma \leq 1, \frac{\varepsilon}{\operatorname{Ro}}=\mathcal{O}(\sqrt{\mu})\right\},
$$

This regime corresponds to a long wave regime $(\varepsilon=\mathcal{O}(\mu))$ under a weak Coriolis forcing $\frac{\varepsilon}{R_{0}}=\mathcal{O}(\sqrt{\mu})$. For an explanation of the first assumption, we refer to [12]. The second assumption is standard in oceanography. Rewriting $\frac{\varepsilon}{\mathrm{Ro}_{0}}=\frac{f L_{x}}{\sqrt{g H}}$, this assumption means that the rotation period is assumed to be much smaller than the time scale of the waves. We refer to [9, 7] for more explanations about this assumption (see also [10, 17, 8, 14]).

We organize this paper in four parts. In Section 1.2, we explain how we nondimensionalize the equations and we provide a local wellposedness result. In Section 2, we derive and justify the Boussinesq-Coriolis equations in the asymptotic regime $\mathcal{A}_{\text {boussi }}$. The Boussinesq-Coriolis equations are a system of three equations on the surface $\zeta$ and the vertical average of the horizontal velocity denoted $\overline{\mathbf{V}}$ (defined in (9)). They correspond to a $\mathcal{O}\left(\mu^{2}\right)$ approximation of the water waves equations. These equations are

$$
\left\{\begin{array}{l}
\partial_{t} \zeta+\nabla^{\gamma} \cdot([1+\varepsilon \zeta] \overline{\mathbf{V}})=0, \\
\left(1-\frac{\mu}{3} \nabla^{\gamma} \nabla^{\gamma}\right) \partial_{t} \overline{\mathbf{V}}+\nabla^{\gamma} \zeta+\varepsilon \overline{\mathbf{V}} \cdot \nabla^{\gamma} \overline{\mathbf{V}}+\frac{\varepsilon}{\mathrm{Ro}} \overline{\mathbf{V}}^{\perp}=0 .
\end{array}\right.
$$

Then, in Section 3, we study the KP approximation which corresponds to the asymptotic regime $\mathcal{A}_{\text {boussi }}$ with $\varepsilon=\mu$ and $\gamma=\sqrt{\mu}$. This second assumption corresponds to weakly transverse effects (see for instance [12]). In this regime, we derive two other asymptotic models. When the Coriolis forcing is weak $\left(\frac{\varepsilon}{R_{0}}=\sqrt{\mu}\right)$, we rigorously justify the modifiedrotation Kadomtsev-Petviashvili equation (see Subsection 3.1), also called GrimshawMelville equation in the physics literature,

$$
\partial_{\xi}\left(\partial_{\tau} k+\frac{3}{2} k \partial_{\xi} k+\frac{1}{6} \partial_{\xi}^{3} k\right)+\frac{1}{2} \partial_{y y} k=\frac{1}{2} k .
$$

Then, when the Coriolis forcing is very weak $\left(\frac{\varepsilon}{R_{0}}=\mu\right)$, we fully justify the KP equation (see Subsection 3.2)

$$
\partial_{\xi}\left(\partial_{\tau} k+\frac{3}{2} k \partial_{\xi} k+\frac{1}{6} \partial_{\xi}^{3} k\right)+\frac{1}{2} \partial_{y y} k=0 .
$$

Finally, in Section 4, we compare the scalar asymptotic models we derive in Section 3 with the ones derived in [16] : the Ostrovsky equation and the KdV equation. 


\subsection{Notations/Definitions}

- If $\mathbf{A} \in \mathbb{R}^{3}$, we denote by $\mathbf{A}_{h}$ its horizontal component.

- If $\mathbf{V}=\left(\begin{array}{l}u \\ v\end{array}\right) \in \mathbb{R}^{2}$, we define the orthogonal of $\mathbf{V}$ by $\mathbf{V}^{\perp}=\left(\begin{array}{c}-v \\ u\end{array}\right)$.

- In this paper, $C(\cdot)$ is a nondecreasing and positive function whose exact value has no importance.

- Consider a vector field $\mathbf{A}$ or a function $\mathrm{w}$ defined on $\Omega$. Then, we denote $\underline{\mathbf{A}}=\mathbf{A}_{\mid z=\varepsilon \zeta}$, $\underline{\mathrm{w}}=\mathrm{w}_{\mid z=\varepsilon \zeta}$ and $\mathbf{A}_{b}=\mathbf{A}_{\mid z=-1}, \mathrm{w}_{b}=\mathrm{w}_{\mid z=-1}$.

- If $N \in \mathbb{N}$ and $f$ is a function on $\mathbb{R}^{2},|f|_{H^{N}}$ is its $H^{N}$-norm, $|f|_{2}$ is its $L^{2}$-norm and $|f|_{L^{\infty}}$ its $L^{\infty}$-norm. We denote by $(,)_{2}$ the $L^{2}\left(\mathbb{R}^{2}\right)$ inner product.

- If $f$ is a function defined on $\mathbb{R}^{2}$, We use the notation $\nabla^{\gamma} f=\left(\partial_{x} f, \gamma \partial_{y} f\right)^{t}$.

- If $u=u(X, z)$ is defined in $\Omega$, we define

$$
\bar{u}(X)=\frac{1}{1+\varepsilon \zeta} \int_{-1}^{\varepsilon \zeta(X)} u(X, z) d z \text { and } u^{*}=u-\bar{u} .
$$

- For $N \geq 0$, we define the Hilbert spaces $\partial_{x} H^{N}\left(\mathbb{R}^{2}\right)$

$$
\partial_{x} H^{N}\left(\mathbb{R}^{2}\right)=\left\{k \in H^{N-1}\left(\mathbb{R}^{2}\right), k=\partial_{x} \tilde{k} \text { with } \tilde{k} \in H^{N}\left(\mathbb{R}^{2}\right)\right\} .
$$

The function $\tilde{k}$ is denoted $\partial_{x}^{-1} k$ in the following.

- Similarly, for $N \geq 0$, we define the Hilbert spaces $\partial_{x}^{2} H^{N}\left(\mathbb{R}^{2}\right)$.

- In the following definition, we recall the notion of consistence (see for instance [12]).

Definition 1.1. We say that the Castro-Lannes equations (7) are consistent of order $\mathcal{O}\left(\mu^{2}\right)$ with a system of equations $S$ for $\zeta$ and $\overline{\mathbf{V}}$ if for any smooth solutions $\left(\zeta, \mathbf{U}_{\|}^{\mu, \gamma}, \boldsymbol{\omega}\right)$ of the Castro-Lannes equations (7), the pair $\left(\zeta, \overline{\mathbf{V}}[\varepsilon \zeta]\left(\mathbf{U}_{\|}^{\mu, \gamma}, \boldsymbol{\omega}\right)\right)$ (defined in (9)) solves $S$ up to a residual of order $\mathcal{O}\left(\mu^{2}\right)$.

\subsection{Nondimensionalization}

We recall the four dimensionless parameters

$$
\mu=\frac{H^{2}}{L_{x}^{2}}, \varepsilon=\frac{a}{H}, \text { Ro }=\frac{a \sqrt{g H}}{H f L_{x}} \text { and } \gamma=\frac{L_{x}}{L_{y}} .
$$

We nondimensionalize the variables and the unknowns. We introduce (see [12] or [15]) 


$$
\left\{\begin{array}{l}
x^{\prime}=\frac{x}{L_{x}}, y^{\prime}=\frac{y}{L_{y}}, z^{\prime}=\frac{z}{H}, \zeta^{\prime}=\frac{\zeta}{a}, t^{\prime}=\frac{\sqrt{g H}}{L_{x}} t \\
\mathbf{V}^{\prime}=\sqrt{\frac{H}{g}} \frac{\mathbf{V}}{a}, \mathrm{w}^{\prime}=H \sqrt{\frac{H}{g}} \frac{\mathrm{w}}{a L_{x}} \text { and } \mathcal{P}^{\prime}=\frac{\mathcal{P}}{\rho g H}
\end{array}\right.
$$

In the following, we use the following notations

$$
\nabla^{\gamma}=\nabla_{X^{\prime}}^{\gamma}=\left(\begin{array}{c}
\partial_{x^{\prime}} \\
\gamma \partial_{y^{\prime}}
\end{array}\right), \nabla_{X^{\prime}, z^{\prime}}^{\mu, \gamma}=\left(\begin{array}{c}
\sqrt{\mu} \nabla_{X^{\prime}}^{\gamma} \\
\partial_{z^{\prime}}
\end{array}\right), \operatorname{curl}^{\mu, \gamma}=\nabla_{X^{\prime}, z^{\prime}}^{\mu, \gamma} \times, \operatorname{div}^{\mu, \gamma}=\nabla_{X^{\prime}, z^{\prime}}^{\mu, \gamma}
$$

We also define

$$
\mathbf{U}^{\mu}=\left(\begin{array}{c}
\sqrt{\mu} \mathbf{V}^{\prime} \\
\mathrm{w}^{\prime}
\end{array}\right), \boldsymbol{\omega}^{\prime}=\frac{1}{\mu} \operatorname{curl}^{\mu, \gamma} \mathbf{U}^{\mu}
$$

and

$$
\underline{\mathbf{U}}^{\mu}=\left(\begin{array}{c}
\sqrt{\mu} \underline{\mathbf{V}}^{\prime} \\
\underline{\underline{\mathrm{w}}^{\prime}}
\end{array}\right)=\mathbf{U}_{\mid z^{\prime}=\varepsilon \zeta^{\prime}}^{\mu}, \mathbf{U}_{b}^{\mu}=\mathbf{U}_{\mid z^{\prime}=-1}^{\mu}, \mathbf{N}^{\mu, \gamma}=\left(\begin{array}{c}
-\varepsilon \sqrt{\mu} \nabla^{\gamma} \zeta^{\prime} \\
1
\end{array}\right) .
$$

Remark 1.2. Notice that the nondimensionalization of the vorticity presented in (6) corresponds to weakly sheared flows (see [3], [20], [18]).

The nondimensionalized fluid domain is

$$
\Omega_{t^{\prime}}^{\prime}:=\left\{\left(X^{\prime}, z^{\prime}\right) \in \mathbb{R}^{3},-1<z^{\prime}<\varepsilon \zeta^{\prime}\left(t^{\prime}, X^{\prime}\right)\right\} .
$$

Finally, the Euler-Coriolis equations (1) become

$$
\left\{\begin{array}{l}
\partial_{t^{\prime}} \mathbf{U}^{\mu}+\frac{\varepsilon}{\mu}\left(\mathbf{U}^{\mu} \cdot \nabla_{X^{\prime}, z^{\prime}}^{\mu, \gamma}\right) \mathbf{U}^{\mu}+\frac{\varepsilon \sqrt{\mu}}{\operatorname{Ro}}\left(\begin{array}{c}
\mathbf{V}^{\prime \perp} \\
0
\end{array}\right)=-\frac{1}{\varepsilon} \nabla_{X^{\prime}, z^{\prime}}^{\mu, \gamma} \mathcal{P}^{\prime}-\frac{1}{\varepsilon} \boldsymbol{e}_{\boldsymbol{z}} \text { in } \Omega_{t}^{\prime} \\
\operatorname{div}_{X^{\prime}, z^{\prime}}^{\mu, \gamma} \mathbf{U}^{\mu}=0 \text { in } \Omega_{t}^{\prime},
\end{array}\right.
$$

with the boundary conditions

$$
\left\{\begin{array}{l}
\partial_{t^{\prime}} \zeta^{\prime}-\frac{1}{\mu} \underline{\mathbf{U}}^{\mu} \cdot \mathbf{N}^{\mu, \gamma}=0 \\
\mathrm{w}_{b}^{\prime}=0
\end{array}\right.
$$

In the following, we omit the primes. We can proceed similarly to nondimensionalize the Castro-Lannes formulation. We define the quantity

$$
\mathbf{U}_{/ /}^{\mu, \gamma}=\underline{\mathbf{V}}+\varepsilon \underline{\mathrm{w}} \nabla^{\gamma} \zeta
$$

Then, the Castro-Lannes formulation becomes (see [4] or [15] when $\gamma=1$ ), 


$$
\left\{\begin{array}{l}
\partial_{t} \zeta-\frac{1}{\mu} \underline{\mathbf{U}}^{\mu} \cdot \mathbf{N}^{\mu, \gamma}=0, \\
\partial_{t} \mathbf{U}_{/ /, \gamma}^{\mu,} \nabla^{\gamma} \zeta+\frac{\varepsilon}{2} \nabla^{\gamma}\left|\mathbf{U}_{/ /}^{\mu, \gamma}\right|^{2}-\frac{\varepsilon}{2 \mu} \nabla^{\gamma}\left[\left(1+\varepsilon^{2} \mu\left|\nabla^{\gamma} \zeta\right|^{2}\right) \underline{\underline{w}}^{2}\right]+\varepsilon\left(\nabla^{\perp} \cdot \mathbf{U}_{/ /}^{\mu, \gamma}\right) \underline{\mathbf{V}}^{\perp}+\frac{\varepsilon}{\mathrm{Ro}} \underline{\mathbf{V}}^{\perp}=0 \\
\partial_{t} \boldsymbol{\omega}+\frac{\varepsilon}{\mu}\left(\mathbf{U}^{\mu} \cdot \nabla_{X, z}^{\mu, \gamma}\right) \boldsymbol{\omega}=\frac{\varepsilon}{\mu}\left(\boldsymbol{\omega} \cdot \nabla_{X, z}^{\mu, \gamma}\right) \mathbf{U}^{\mu}+\frac{\varepsilon}{\mu \mathrm{Ro}} \partial_{z} \mathbf{U}^{\mu},
\end{array}\right.
$$

where $\mathbf{U}^{\mu}=\left(\begin{array}{c}\sqrt{\mu} \mathbf{V} \\ \mathrm{w}\end{array}\right)=\mathbf{U}^{\mu}[\varepsilon \zeta]\left(\mathbf{U}_{/ /}^{\mu, \gamma}, \boldsymbol{\omega}\right)$ is the unique solution in $H^{1}\left(\Omega_{t}\right)$ of

$$
\left\{\begin{array}{l}
\operatorname{curl}^{\mu, \gamma} \mathbf{U}^{\mu}=\mu \boldsymbol{\omega} \text { in } \Omega_{t}, \\
\operatorname{div}^{\mu, \gamma} \mathbf{U}^{\mu}=0 \text { in } \Omega_{t}, \\
\left(\underline{\mathbf{V}}+\varepsilon \underline{\mathbf{w}} \nabla^{\gamma} \zeta\right)_{\mid z=\varepsilon \zeta}=\mathbf{U}_{/ /}^{\mu, \gamma}, \\
\mathbf{w}_{b}=0 .
\end{array}\right.
$$

In order to rigorously derive asymptotic models, we need an existence result for the Castro-Lannes formulation (77). We recall that the existence of solutions to the water waves equations is always obtained under the so-called Rayleigh-Taylor condition that assumes the positivity of the Rayleigh-Taylor coefficient $\mathfrak{a}$ (see Part 3.4.5 in [12] for the link between $\mathfrak{a}$ and the Rayleigh-Taylor condition or [15]) where

$$
\mathfrak{a}:=\mathfrak{a}[\varepsilon \zeta]\left(\mathbf{U}_{/ /}^{\mu, \gamma}, \boldsymbol{\omega}\right)=1+\varepsilon\left(\partial_{t}+\varepsilon \underline{\mathbf{V}}[\varepsilon \zeta]\left(\mathbf{U}_{/ /}^{\mu, \gamma}, \boldsymbol{\omega}\right) \cdot \nabla\right) \underline{\mathrm{w}}[\varepsilon \zeta]\left(\mathbf{U}_{/ /}^{\mu, \gamma}, \boldsymbol{\omega}\right) .
$$

We explain in [15] how we can define the Rayleigh-Taylor coefficient $\mathfrak{a}$ at $t=0$. We also assume that the water depth is bounded from below by a positive constant

$$
\exists h_{\text {min }}>0,1+\varepsilon \zeta \geq h_{\text {min }} .
$$

The following theorem can be found in [15] and provide a local wellposedness result of the Castro-Lannes formulation (7) (see also Theorem 1.5 in [16]).

Theorem 1.3. Let $A>0$ and $\mathbf{N} \geq 5$. We suppose that $(\mu, \varepsilon, \gamma, \operatorname{Ro}) \in \mathcal{A}_{\text {boussi }}$. We assume that

$$
\left(\zeta_{0},\left(\mathbf{U}_{\|}^{\mu, \gamma}\right)_{0}, \boldsymbol{\omega}_{0}\right) \in H^{N+\frac{1}{2}}\left(\mathbb{R}^{2}\right) \times H^{N}\left(\mathbb{R}^{2}\right) \times H^{N-1}\left(\Omega_{0}\right)
$$

with $\nabla^{\mu, \gamma} \cdot \boldsymbol{\omega}_{0}=0$ and $\nabla^{\gamma \perp} \cdot\left(\mathbf{U}_{\|}^{\mu, \gamma}\right)_{0}=\underline{\boldsymbol{\omega}_{0}} \cdot\left(\begin{array}{c}-\varepsilon \sqrt{\mu} \nabla^{\gamma} \zeta_{0} \\ 1\end{array}\right)$. Finally, we assume that

$$
\exists h_{\min }, \mathfrak{a}_{\min }>0,1+\varepsilon \zeta_{0} \geq h_{\min } \text { and } \mathfrak{a}\left[\varepsilon \zeta_{0}\right]\left(\left(\mathbf{U}_{\|}^{\mu, \gamma}\right)_{0}, \boldsymbol{\omega}_{0}\right) \geq \mathfrak{a}_{\min },
$$

and that

$$
\left|\zeta_{0}\right|_{H^{N+\frac{1}{2}}}+\left|\frac{1}{\sqrt{1+\sqrt{\mu}|D|}}\left(\mathbf{U}_{/ /}^{\mu, \gamma}\right)_{0}\right|_{H^{N}}+\left.|| \boldsymbol{\omega}_{0}\right|_{H^{N-1}} \leq A
$$


Then, there exists $T>0$ and a unique classical solution $\left(\zeta, \mathbf{U}_{\|}^{\mu, \gamma}, \boldsymbol{\omega}\right)$ to the CastroLannes (7) on $[0, T]$ with initial data $\left(\zeta_{0},\left(\mathbf{U}_{\|}^{\mu, \gamma}\right)_{0}, \boldsymbol{\omega}_{0}\right)$. Moreover,

$$
\begin{aligned}
& T=\frac{T_{0}}{\max \left(\varepsilon, \frac{\varepsilon}{\mathrm{Ro}}\right)}, \frac{1}{T_{0}}=c^{1}, \\
& \max _{[0, T]}\left(|\zeta(t, \cdot)|_{H^{N}}+\left|\frac{1}{\sqrt{1+\sqrt{\mu}|D|}} \mathbf{U}_{/ /}^{\mu, \gamma}(t, \cdot)\right|_{H^{N-\frac{1}{2}}}+\|\left.\boldsymbol{\omega}(t, \cdot)\right|_{H^{N-1}}\right)=c^{2},
\end{aligned}
$$

with $c^{j}=C\left(A, \mu_{0}, \frac{1}{h_{\min }}, \frac{1}{\mathfrak{a}_{\min }}\right)$.

Remark 1.4. Notice that thanks to Theorem 1.3 together with Part 5.5.1 in [4], the quantities $\zeta, \mathbf{U}_{\|}^{\mu, \gamma}, \boldsymbol{\omega}, \overline{\mathbf{V}}, \mathbf{U}, \partial_{t} \zeta, \partial_{t} \mathbf{U}_{\|}^{\mu, \gamma}, \partial_{t} \boldsymbol{\omega}$ and $\partial_{t} \mathbf{U}$ remain bounded uniformly with respect to the small parameters during the time evolution of the flow.

\section{The Boussinesq-Coriolis equations}

In this part, we derive and fully justify the Boussinesq-Coriolis equations (3) under a weak Coriolis forcing $\frac{\varepsilon}{R_{0}}=\mathcal{O}(\sqrt{\mu})$. We recall the corresponding asymptotic regime

$$
\mathcal{A}_{\text {boussi }}=\left\{(\mu, \varepsilon, \gamma, \operatorname{Ro}), 0 \leq \mu \leq \mu_{0}, \varepsilon=\mathcal{O}(\mu), \gamma \leq 1, \frac{\varepsilon}{\operatorname{Ro}}=\mathcal{O}(\sqrt{\mu})\right\} .
$$

Notice that no assumption on $\gamma$ is made in this part. The Boussinesq equations correspond to an order $\mathcal{O}\left(\mu^{2}\right)$ approximation of the water waves equations. Motivated by [16], we use the Castro-Lannes equations (17) to derive this asymptotic model. We introduce the water depth

$$
h(t, X)=1+\varepsilon \zeta(t, X)
$$

and the vertical average of the horizontal velocity

$$
\overline{\mathbf{V}}=\overline{\mathbf{V}}[\varepsilon \zeta]\left(\mathbf{U}_{\|}^{\mu, \gamma}, \boldsymbol{\omega}\right)(t, X)=\frac{1}{h(t, X)} \int_{z=-1}^{\varepsilon \zeta(t, X)} \mathbf{V}[\varepsilon \zeta, \beta b]\left(\mathbf{U}_{\|}^{\mu, \gamma}, \boldsymbol{\omega}\right)(t, X, z) d z
$$

In the following we denote $\mathbf{V}=(u, v)^{t}$. More generally, if $u$ is a function defined in $\Omega$, we denote by $\bar{u}$ its vertical average and $u^{*}=u-\bar{u}$. We also have to introduce the "shear" velocity

$$
\mathbf{V}_{\mathrm{sh}}=\mathbf{V}_{\mathrm{sh}}[\varepsilon \zeta]\left(\mathbf{U}_{\|}^{\mu, \gamma}, \boldsymbol{\omega}\right)(t, X)=\int_{z}^{\varepsilon \zeta} \boldsymbol{\omega}_{h}^{\perp}\left(t, X, z^{\prime}\right) d z^{\prime}
$$

and its vertical average 


$$
\mathbf{Q}=\overline{\mathbf{V}_{\mathrm{sh}}}=\frac{1}{h} \int_{-1}^{\varepsilon \zeta} \int_{z^{\prime}}^{\varepsilon \zeta} \boldsymbol{\omega}_{h}^{\perp}
$$

As noticed in [4, these quantities appear when one wants to obtain an expansion with respect to $\mu$ of the velocity. We recall that

$$
\mathbf{U}_{\|}^{\mu, \gamma}=\underline{\mathbf{V}}+\varepsilon \underline{\mathrm{w}} \nabla^{\gamma} \zeta
$$

\subsection{Asymptotic expansions with respect to $\mu$}

In this part, we give an expansion of different quantities with respect to $\mu$. These expansions will help us to derive the Boussinesq-Coriolis equations (3) in Section 2.2. The following proposition gives a link between $\overline{\mathbf{V}}$ and $\underline{\mathbf{U}}^{\mu} \cdot \mathbf{N}^{\mu, \gamma}$ (the proof is a small adaptation of Proposition 4.2 in [15]).

Proposition 2.1. If $\left(\zeta, \mathbf{U}_{\| /}^{\mu, \gamma}, \boldsymbol{\omega}\right)$ satisfy the Castro-Lannes system (7), we have

$$
\underline{\mathbf{U}}^{\mu} \cdot \mathbf{N}^{\mu, \gamma}=-\mu \nabla^{\gamma} \cdot(h \overline{\mathbf{V}}) .
$$

Then we get the first equation of the Boussinesq-Coriolis system from the first equation of (7). We also need an expansion of $\mathbf{V}$ and $\mathrm{w}$ with respect to $\mu$. We introduce the following operators

$$
T[\varepsilon \zeta] f=\int_{z}^{\varepsilon \zeta} \nabla^{\gamma} \nabla^{\gamma} \cdot \int_{-1}^{z^{\prime}} f \text { and } T^{*}[\varepsilon \zeta] f=(T[\varepsilon \zeta] f)^{*},
$$

In the following, we denote $T=T[\varepsilon \zeta]$ and $T^{*}=T^{*}[\varepsilon \zeta]$ when no confusion is possible.

Proposition 2.2. In the Boussinesq regime $\mathcal{A}_{\text {boussi }}$, if $\left(\zeta, \mathbf{U}_{\|}^{\mu, \gamma}, \boldsymbol{\omega}\right)$ satisfy the CastroLannes system (7), we have

$$
\begin{aligned}
& \mathbf{V}=\overline{\mathbf{V}}+\sqrt{\mu} \mathbf{V}_{\mathrm{sh}}^{*}+\mu T^{*} \overline{\mathbf{V}}+\mu^{\frac{3}{2}} T^{*} \mathbf{V}_{\mathrm{sh}}^{*}+\mathcal{O}\left(\mu^{2}\right), \\
& \underline{\mathbf{V}}=\overline{\mathbf{V}}-\sqrt{\mu} \mathbf{Q}+\mu \underline{T^{*} \overline{\mathbf{V}}}-\mu^{\frac{3}{2}} \overline{T \mathbf{V}_{\mathrm{sh}}^{*}}+\mathcal{O}\left(\mu^{2}\right),
\end{aligned}
$$

where

$$
T^{*} \overline{\mathbf{V}}=\frac{1}{2}\left(\frac{h^{2}}{3}-[z+1]^{2}\right) \nabla^{\gamma} \nabla^{\gamma} \cdot \overline{\mathbf{V}} \text { and } \underline{T^{*} \overline{\mathbf{V}}}=-\frac{h^{2}}{3} \nabla^{\gamma} \nabla^{\gamma} \cdot \overline{\mathbf{V}}
$$

We also have

$$
\begin{aligned}
& \mathrm{w}=-\mu(z+1) \nabla^{\gamma} \overline{\mathbf{V}}+\mu^{\frac{3}{2}} \int_{-1}^{z} \nabla^{\gamma} \cdot \mathbf{V}_{\mathrm{sh}}^{*}+\mathcal{O}\left(\mu^{2}\right), \\
& \underline{\mathrm{w}}=-\mu h \nabla^{\gamma} \cdot \overline{\mathbf{V}}+\mathcal{O}\left(\mu^{2}\right),
\end{aligned}
$$


Proof. This proof is an adaptation of part 2.2 in [3, Part 4.2 in [15] and Section 2.1 in [16]. First, using $\operatorname{curl}^{\mu, \gamma} \mathbf{U}^{\mu}=\mu \boldsymbol{\omega}$, we obtain that

$$
\sqrt{\mu} \boldsymbol{\omega}_{h}=\partial_{z} \mathbf{V}^{\perp}-\nabla^{\gamma \perp} \mathrm{w} .
$$

Then, we consider the ansatz $\mathbf{V}=\overline{\mathbf{V}}+\sqrt{\mu} \mathbf{V}_{1}$. By integrating the previous equation, we obtain

$$
\sqrt{\mu} \partial_{z} \mathbf{V}_{1}=-\sqrt{\mu} \boldsymbol{\omega}_{h}^{\perp}+\nabla^{\gamma \perp} \mathrm{w}
$$

Since $\overline{\mathbf{V}_{1}}=0$, we get

$$
\mathbf{V}_{1}=\left(\int_{z}^{\varepsilon \zeta} \boldsymbol{\omega}_{h}^{\perp}\right)^{*}-\frac{1}{\sqrt{\mu}}\left(\int_{z}^{\varepsilon \zeta} \nabla^{\gamma} \mathrm{w}\right)^{*}
$$

Secondly, using Proposition 2.1 and the divergence-free assumption, we get

$$
\mathrm{w}=-\mu \nabla^{\gamma} \cdot\left(\int_{-1}^{z} \mathbf{V}\right)
$$

Then, gathering the previous two equality, we obtain

$$
\mathbf{V}=\overline{\mathbf{V}}+\sqrt{\mu} \mathbf{V}_{\mathrm{sh}}^{*}+\mu T^{*} \mathbf{V}
$$

Finally, the expansion of $\mathbf{V}$ follows by applying the operator $I d+\mu T^{*}$ to the previous equality. For the second equality, we notice that $T^{*} \mathbf{V}_{\mathrm{sh}}^{*}=-\overline{T \mathbf{V}_{\mathrm{sh}}^{*}}$. The third and fourth equalities follow from the fact that $\overline{\mathbf{V}}$ does not depend on $z$. The fifth equality are a consequence of Equalities (10) and (11). Finally, the sixth equality follows from the fact that $\overline{\mathbf{V}_{\mathrm{sh}}^{*}}=0$ and that $\varepsilon=\mathcal{O}(\mu)$.

We can also get an expansion of $\partial_{t} \mathbf{V}$ and $\partial_{t} \mathrm{w}$.

Proposition 2.3. In the Boussinesq regime $\mathcal{A}_{\text {boussi }}$, if $\left(\zeta, \mathbf{U}_{\|}^{\mu, \gamma}, \boldsymbol{\omega}\right)$ satisfy the CastroLannes system (7), we have

$$
\begin{aligned}
& \partial_{t}\left(\mathbf{V}-\overline{\mathbf{V}}-\sqrt{\mu} \mathbf{V}_{\mathrm{sh}}^{*}-\mu T^{*} \overline{\mathbf{V}}-\mu^{\frac{3}{2}} T^{*} \mathbf{V}_{\mathrm{sh}}^{*}\right)=\mathcal{O}\left(\mu^{2}\right), \\
& \partial_{t}\left(\underline{\mathbf{V}}-\overline{\mathbf{V}}+\sqrt{\mu} \mathbf{Q}-\mu \underline{T^{*} \overline{\mathbf{V}}}+\mu^{\frac{3}{2}} \overline{T \mathbf{V}_{\mathrm{sh}}^{*}}\right)=\mathcal{O}\left(\mu^{2}\right), \\
& \partial_{t}\left(\underline{\mathbf{w}}+\mu h \nabla^{\gamma} \overline{\mathbf{V}}\right)=\mathcal{O}\left(\mu^{2}\right) .
\end{aligned}
$$

Proof. The result follows from Proposition 2.1 and the equality

$$
\mathbf{V}=\left(1-\mu T^{*}\right)\left(\overline{\mathbf{V}}+\sqrt{\mu} \mathbf{V}_{\mathrm{sh}}^{*}\right)+\mu^{2} T^{*} T^{*} \mathbf{V}
$$

Since we can not express $\mathrm{Q}$ and $\mathbf{V}_{\text {sh }}^{*}$ with respect to $\zeta$ and $\overline{\mathbf{V}}$, we need an evolution equation at order $\mathcal{O}\left(\mu^{\frac{3}{2}}\right)$ of these quantities. 
Proposition 2.4. In the Boussinesq regime $\mathcal{A}_{\text {boussi }}$, if $\left(\zeta, \mathbf{U}_{\|}^{\mu, \gamma}, \boldsymbol{\omega}\right)$ satisfy the CastroLannes system (7), then $Q$ satisfies the following equation

$$
\partial_{t} \mathbf{Q}+\varepsilon \overline{\mathbf{V}} \cdot \nabla^{\gamma} \mathbf{Q}+\varepsilon \mathbf{Q} \cdot \nabla^{\gamma} \overline{\mathbf{V}}+\frac{\varepsilon}{\operatorname{Ro} \sqrt{\mu}}(\overline{\mathbf{V}}-\underline{\mathbf{V}})^{\perp}=\mathcal{O}\left(\mu^{\frac{3}{2}}\right)
$$

and $\boldsymbol{V}_{\text {sh }}^{*}$ satisfies the equation

$$
\partial_{t} \mathbf{V}_{\mathrm{sh}}^{*}+\varepsilon \overline{\mathbf{V}} \cdot \nabla^{\gamma} \mathbf{V}_{\mathrm{sh}}^{*}+\varepsilon \mathbf{V}_{\mathrm{sh}}^{*} \cdot \nabla^{\gamma} \overline{\mathbf{V}}-\varepsilon[1+z]\left(\nabla^{\gamma} \cdot \overline{\mathbf{V}}\right) \partial_{z} \mathbf{V}_{\mathrm{sh}}^{*}+\frac{\varepsilon}{\operatorname{Ro} \sqrt{\mu}}(\mathbf{V}-\overline{\mathbf{V}})^{\perp}=\mathcal{O}\left(\mu^{\frac{3}{2}}\right)
$$

Proof. This proof is an adaptation of Part 2.3 in [3] and Part 2.2 in [16. Thanks to the horizontal component of the vorticity equation of the Castro-Lannes formulation (7), we get

$$
\partial_{t} \boldsymbol{\omega}_{h}+\varepsilon \mathbf{V} \cdot \nabla^{\gamma} \boldsymbol{\omega}_{h}+\frac{\varepsilon}{\mu} \mathrm{w} \partial_{z} \boldsymbol{\omega}_{h}=\varepsilon \boldsymbol{\omega}_{h} \cdot \nabla^{\gamma} \mathbf{V}+\frac{\varepsilon}{\sqrt{\mu}} \boldsymbol{\omega}_{z} \partial_{z} \mathbf{V}+\frac{\varepsilon}{\operatorname{Ro} \sqrt{\mu}} \partial_{z} \mathbf{V}
$$

Furthermore, since $\operatorname{curl}^{\mu, \gamma} \mathbf{U}^{\mu}=\mu \boldsymbol{\omega}$, we have

$$
\partial_{z} \mathbf{V}=-\sqrt{\mu} \boldsymbol{\omega}_{h}^{\perp}+\mathcal{O}(\mu) \text { and } \boldsymbol{\omega}_{z}=\nabla^{\gamma \perp} \cdot \overline{\mathbf{V}}+\mathcal{O}(\sqrt{\mu})
$$

Then, using Proposition 2.2, we obtain

$\partial_{t} \boldsymbol{\omega}_{h}+\varepsilon \overline{\mathbf{V}} \cdot \nabla^{\gamma} \boldsymbol{\omega}_{h}-\varepsilon[1+z]\left(\nabla^{\gamma} \cdot \overline{\mathbf{V}}\right) \partial_{z} \boldsymbol{\omega}_{h}-\varepsilon \boldsymbol{\omega}_{h} \cdot \nabla^{\gamma} \overline{\mathbf{V}}-\varepsilon\left(\nabla^{\gamma \perp} \overline{\mathbf{V}}\right) \boldsymbol{\omega}_{h}^{\perp}-\frac{\varepsilon}{\operatorname{Ro} \sqrt{\mu}} \partial_{z} \mathbf{V}=\mathcal{O}\left(\mu^{\frac{3}{2}}\right)$,

Then, integrating with respect to $z$, using the fact that $\partial_{t} \zeta+\nabla^{\gamma} \cdot(h \overline{\mathbf{V}})=0, \mathbf{V}_{\mathrm{sh}}=\int_{z}^{\varepsilon \zeta} \boldsymbol{\omega}_{h}^{\perp}$ and $\mathbf{Q}_{x}=\overline{\mathbf{V}_{\mathrm{sh}}^{*}}$ we get (see the computations in Part 2.3 in [3]

$\partial_{t} \mathbf{V}_{\mathrm{sh}}+\varepsilon \overline{\mathbf{V}} \cdot \nabla^{\gamma} \mathbf{V}_{\mathrm{sh}}+\varepsilon \mathbf{V}_{\mathrm{sh}} \cdot \nabla^{\gamma} \overline{\mathbf{V}}+\frac{\varepsilon}{\operatorname{Ro} \sqrt{\mu}}(\mathbf{V}-\underline{\mathbf{V}})^{\perp}=\varepsilon[1+z]\left(\nabla^{\gamma} \cdot \overline{\mathbf{V}}\right) \partial_{z} \mathbf{V}_{\mathrm{sh}}+\mathcal{O}\left(\mu^{\frac{3}{2}}\right)$ and

$$
\partial_{t} \mathbf{Q}+\varepsilon \overline{\mathbf{V}} \cdot \nabla^{\gamma} \mathbf{Q}+\varepsilon \mathbf{Q} \cdot \nabla^{\gamma} \overline{\mathbf{V}}+\frac{\varepsilon}{\operatorname{Ro} \sqrt{\mu}}(\overline{\mathbf{V}}-\underline{\mathbf{V}})^{\perp}=\mathcal{O}\left(\mu^{\frac{3}{2}}\right)
$$

Finally, the second equation follows from the fact that $\mathbf{V}_{\mathrm{sh}}^{*}=\mathbf{V}_{\mathrm{sh}}-\mathbf{Q}$.

\subsection{Full justification of the Boussinesq-Coriolis equations}

We can now establish the Boussinesq-Coriolis equations under a weak Coriolis forcing. The Boussinesq-Coriolis equations (3) are the following system

$$
\left\{\begin{array}{l}
\partial_{t} \zeta+\nabla^{\gamma} \cdot h \overline{\mathbf{V}}=0, \\
\left(1-\frac{\mu}{3} \nabla^{\gamma} \nabla^{\gamma} \cdot\right) \partial_{t} \overline{\mathbf{V}}+\nabla^{\gamma} \zeta+\varepsilon \overline{\mathbf{V}} \cdot \nabla^{\gamma} \overline{\mathbf{V}}+\frac{\varepsilon}{\mathrm{Ro}_{\mathrm{o}}} \overline{\mathbf{V}}^{\perp}=0 .
\end{array}\right.
$$

First, we show a consistency result. 
Proposition 2.5. In the Boussinesq regime $\mathcal{A}_{\text {Boussi }}$, the Castro-Lannes equations (7) are consistent at order $\mathcal{O}\left(\mu^{2}\right)$ with the Boussinesq-Coriolis equations (3) in the sense of Definition 1.1.

Proof. The first equation of the Boussinesq-Coriolis equations is always satisfied for a solution of the Castro-Lannes formulation by Proposition 2.1. We recall that the second equation of the Castro-Lannes formulation is

$$
\partial_{t} \mathbf{U}_{\|}^{\mu, \gamma}+\nabla^{\gamma} \zeta+\frac{\varepsilon}{2} \nabla^{\gamma}\left|\mathbf{U}_{\|}^{\mu, \gamma}\right|^{2}-\frac{\varepsilon}{2 \mu} \nabla^{\gamma}\left[\left(1+\varepsilon^{2} \mu\left|\nabla^{\gamma} \zeta\right|^{2}\right) \underline{\mathrm{w}}^{2}\right]+\varepsilon\left(\nabla^{\perp} \cdot \mathbf{U}_{\|}^{\mu, \gamma}\right) \underline{\mathbf{V}}^{\perp}+\frac{\varepsilon}{\mathrm{Ro}} \underline{\mathbf{V}}^{\perp}=0
$$

Thanks to Proposition 2.2, we know that $(\varepsilon=\mathcal{O}(\mu))$

$$
\mathbf{U}_{\|}^{\mu, \gamma}=\underline{\mathbf{V}}+\varepsilon \underline{\mathrm{w}} \nabla^{\gamma} \zeta=\underline{\mathbf{V}}+\mathcal{O}\left(\mu^{2}\right)=\overline{\mathbf{V}}-\sqrt{\mu} \mathbf{Q}+\mu \underline{T^{*} \overline{\mathbf{V}}}-\mu^{\frac{3}{2}} \overline{T \mathbf{V}_{\mathrm{sh}}^{*}}+\mathcal{O}\left(\mu^{2}\right),
$$

and

$$
\begin{aligned}
& \frac{\varepsilon}{2} \nabla^{\gamma}\left|\mathbf{U}_{\|}^{\mu, \gamma}\right|^{2}=\varepsilon \mathbf{U}_{\|}^{\mu, \gamma} \cdot \nabla^{\gamma} \mathbf{U}_{\|}^{\mu, \gamma}-\varepsilon\left(\nabla^{\gamma \perp} \cdot \mathbf{U}_{\|}^{\mu, \gamma}\right) \mathbf{U}_{\|}^{\mu, \gamma \perp} \\
& =\varepsilon \overline{\mathbf{V}} \cdot \nabla^{\gamma} \overline{\mathbf{V}}-\varepsilon \sqrt{\mu} \mathbf{Q} \cdot \nabla^{\gamma} \overline{\mathbf{V}}-\varepsilon \sqrt{\mu \overline{\mathbf{V}}} \cdot \nabla^{\gamma} \mathbf{Q}-\varepsilon\left(\nabla^{\gamma \perp} \cdot \mathbf{U}_{\|}^{\mu, \gamma}\right) \underline{\mathbf{V}}^{\perp}+\mathcal{O}\left(\mu^{2}\right) .
\end{aligned}
$$

Furthermore, thanks to Proposition 2.4 and Proposition 2.2, we get $\left(\frac{\varepsilon}{\mathrm{Ro}}=\mathcal{O}(\sqrt{\mu})\right)$

$$
\mu^{\frac{3}{2}} \partial_{t} \overline{T \mathbf{V}_{\mathrm{sh}}^{*}}=\mu^{\frac{3}{2}} \overline{T \partial_{t} \mathbf{V}_{\mathrm{sh}}^{*}}+\mathcal{O}\left(\mu^{2}\right)=-\mu^{\frac{3}{2}} \frac{\varepsilon}{\mathrm{Ro}} \overline{T \mathbf{V}_{\mathrm{sh}}^{* \perp}}+\mathcal{O}\left(\mu^{2}\right)=\mathcal{O}\left(\mu^{2}\right)
$$

Finally, using Proposition 2.2, Proposition 2.4, Proposition 2.3 and Remark 1.4, we obtain from the second equation of the Castro-Lannes formulation that

$$
\left(1-\frac{\mu}{3} \nabla^{\gamma} \nabla^{\gamma} \cdot\right) \partial_{t} \overline{\mathbf{V}}+\nabla^{\gamma} \zeta+\varepsilon \overline{\mathbf{V}} \cdot \nabla^{\gamma} \overline{\mathbf{V}}+\frac{\varepsilon}{\mathrm{Ro}} \overline{\mathbf{V}}^{\perp}=\mathcal{O}\left(\mu^{2}\right)
$$

Notice that all the terms that involve $\mathbf{Q}$ disappear (this fact was pointed out in [3] and [15]).

Remark 2.6. In [16], the author points out the fact that under a strong Coriolis forcing $\left(\frac{\varepsilon}{R o} \leq 1\right)$, a new term appears in the Boussinesq-Coriolis equations. We would like to emphasize that this term is not present in this setting since we only study a weak Coriolis forcing $\left(\frac{\varepsilon}{R o}=\mathcal{O}(\sqrt{\mu})\right)$.

The purpose of this part is to fully justify the Boussinesq-Coriolis equations (3). First, we give a local wellposedness result of the Boussinesq-Coriolis equations. We define the energy space

$$
X_{\mu}^{N}\left(\mathbb{R}^{2}\right)=H^{N}\left(\mathbb{R}^{2}\right) \times H^{N}\left(\mathbb{R}^{2}\right) \times H^{N}\left(\mathbb{R}^{2}\right),
$$

endowed with the norm

$$
|(\zeta, \mathbf{V})|_{X_{\mu}^{N}}^{2}=|\zeta|_{H^{N}}^{2}+|\mathbf{V}|_{H^{N}}^{2}+\mu\left|\nabla^{\gamma} \cdot \mathbf{V}\right|_{H^{N}}^{2}
$$


Proposition 2.7. Let $N \geq 3$ and $\left(\zeta_{0}, \overline{\mathbf{V}}_{0}\right) \in X_{\mu}^{N}\left(\mathbb{R}^{2}\right)$. Suppose that $(\mu, \varepsilon, \gamma, \operatorname{Ro}) \in$ $\mathcal{A}_{\text {boussi. }}$. Assume that

$$
\exists h_{\min }>0,1+\varepsilon \zeta_{0} \geq h_{\min } .
$$

Then, there exists an existence time $T>0$ and a unique solution $(\zeta, \overline{\mathbf{V}})$ on $[0, T]$ to the Boussinesq-Coriolis equations (3) with initial data $\left(\zeta_{0}, \overline{\mathbf{V}}_{0}\right)$. Moreover, $(\zeta, \overline{\mathbf{V}}) \in$ $\mathcal{C}\left([0, T] ; X_{\mu}^{N}\left(\mathbb{R}^{2}\right)\right)$ and

$$
T=\frac{T_{0}}{\mu}, \frac{1}{T_{0}}=c^{1} \text { and } \max _{[0, T]}|(\zeta, \overline{\mathbf{V}})(t, \cdot)|_{X_{\mu}^{N}}=c^{2},
$$

with $c^{j}=C\left(\mu_{0}, \frac{1}{h_{\min }},\left|\left(\zeta_{0}, \overline{\mathbf{V}}_{0}\right)\right|_{X_{\mu}^{N}}\right)$.

Proof. This proof is a small adaptation of the proof of Proposition 6.7 in [12]. We only give the energy estimates. We assume that $(\zeta, \overline{\mathbf{V}})$ solves (3) on $\left[0, \frac{T_{0}}{\mu}\right]$ and that

$$
1+\varepsilon \zeta \geq \frac{h_{\min }}{2} \text { on }\left[0, \frac{T_{0}}{\mu}\right] .
$$

We denote $U=(\zeta, \overline{\mathbf{V}})^{t}$. We introduce the symmetric matrix operator

$$
S(U)=\left(\begin{array}{cc}
1 & 0 \\
0 & h I_{2}-\mu \frac{1}{3} \nabla^{\gamma}\left(h \nabla^{\gamma} \cdot\right)
\end{array}\right)
$$

and the associated energy

$$
\mathcal{E}^{N}(U)=\frac{1}{2} \sum_{|\alpha| \leq N}\left(S(U) \partial^{\alpha} U, \partial^{\alpha} U\right)_{2} .
$$

Remark that there exists $c_{1}, c_{2}=C\left(\frac{1}{h_{\min }},|h|_{L^{\infty}}\right)$ such that

$$
c_{1}\left|\nabla^{\gamma} \cdot \mathbf{V}\right|_{2}^{2} \leq\left(-\frac{1}{3} \nabla^{\gamma}\left(h \nabla^{\gamma} \cdot \overline{\mathbf{V}}\right), \overline{\mathbf{V}}\right)_{2} \leq c_{2}\left|\nabla^{\gamma} \cdot \mathbf{V}\right|_{2}^{2} .
$$

We also notice that for $|\alpha|=N$,

$$
\frac{d}{d t}\left(S(U) \partial^{\alpha} U\right)=\left(\begin{array}{c}
\partial^{\alpha} \partial_{t} \zeta \\
h\left(1-\frac{\mu}{3} \nabla^{\gamma} \nabla^{\gamma} .\right) \partial^{\alpha} \partial_{t} \overline{\mathbf{V}}
\end{array}\right)-\left(\begin{array}{c}
0 \\
\varepsilon \frac{\mu}{3}\left(\nabla^{\gamma} \cdot \partial^{\alpha} \partial_{t} \overline{\mathbf{V}}\right) \nabla^{\gamma} \zeta
\end{array}\right)+\varepsilon \text { l.o.t. }
$$

and that, denoting $\Delta^{\gamma}=\nabla^{\gamma} \cdot \nabla^{\gamma}$,

$$
\mu\left|\nabla^{\gamma} \cdot \partial_{t} \overline{\mathbf{V}}\right|_{H^{N}} \leq\left|\left(1-\frac{\mu}{3} \Delta^{\gamma}\right)^{-1} \mu \nabla^{\gamma} \cdot\left(\nabla^{\gamma} \zeta+\varepsilon \overline{\mathbf{V}} \cdot \nabla^{\gamma} \overline{\mathbf{V}}+\frac{\varepsilon}{\operatorname{Ro}} \overline{\mathbf{V}}^{\perp}\right)\right|_{H^{N}} \leq C\left(\mu_{0}, \mathcal{E}^{N}(U)\right)
$$

Then, after some computations we obtain $(\varepsilon=\mathcal{O}(\mu))$ 


$$
\frac{d}{d t} \mathcal{E}^{N}(U) \leq \mu C\left(\mathcal{E}^{N}(U)\right) \mathcal{E}^{N}(U)
$$

and the result follows from Grönwall's inequality.

We also have a stability result for the Boussinesq-Coriolis equations (3).

Proposition 2.8. Let the assumptions of Proposition 2.7 be satisfied. Suppose that there exists $(\tilde{\zeta}, \tilde{\mathbf{V}}) \in \mathcal{C}\left(\left[0, \frac{T_{0}}{\mu}\right] ; X_{\mu}^{N}\left(\mathbb{R}^{2}\right)\right)$ satisfying

$$
\left\{\begin{array}{l}
\partial_{t} \tilde{\zeta}+\nabla^{\gamma} \cdot \tilde{h} \tilde{\mathbf{V}}=R_{1}, \\
\left(1-\frac{\mu}{3} \nabla^{\gamma} \nabla^{\gamma}\right) \partial_{t} \tilde{\mathbf{V}}+\nabla^{\gamma} \tilde{\zeta}+\varepsilon \tilde{\mathbf{V}} \cdot \nabla^{\gamma} \tilde{\mathbf{V}}+\frac{\varepsilon}{\operatorname{Ro}} \tilde{\mathbf{V}}^{\perp}=R_{2} .
\end{array}\right.
$$

where $\tilde{h}=1+\varepsilon \tilde{\zeta}$ and with $R=\left(R_{1}, R_{2}\right) \in L^{\infty}\left(\left[0, \frac{T_{0}}{\mu}\right] ; H^{N-1}\left(\mathbb{R}^{2}\right)\right)$. Then, if we denote $\mathfrak{e}=(\zeta, \overline{\mathbf{V}})-(\tilde{\zeta}, \tilde{\mathbf{V}})$ where $U=(\zeta, \mathbf{V})$ is the solution given in Proposition 2.7, we have

$$
|\mathfrak{e}(t)|_{X_{\mu}^{N-1}} \leq c_{1}\left(\left|\mathfrak{e}_{\mid t=0}\right|_{X_{\mu}^{N-1}}+t|R|_{L^{\infty}}\left(\left[0, \frac{T_{0}}{\mu}\right] ; H^{N-1}\right)\right)
$$

where

$$
c_{1}=C\left(\mu_{0}, \frac{1}{h_{\min }},\left|\left(\zeta_{0}, \overline{\mathbf{V}}_{0}\right)\right|_{X_{\mu}^{N}},|(\tilde{\zeta}, \tilde{\mathbf{V}})|_{L^{\infty}\left(\left[0, \frac{T_{0}}{\mu}\right] ; X_{\mu}^{N}\right)},|R|_{L^{\infty}\left(\left[0, \frac{T_{0}}{\mu}\right] ; H^{N-1}\right)}\right)
$$

Proof. We proceed as in Proposition 2.7. We define the energy

$$
\mathcal{F}^{N-1}(\mathfrak{e})=\frac{1}{2} \sum_{|\alpha| \leq N-1}\left(S(U) \partial^{\alpha} \mathfrak{e}, \partial^{\alpha} \mathfrak{e}\right)_{2}
$$

After some computations, we get

$$
\frac{d}{d t} \mathcal{F}^{N-1}(\mathfrak{e}) \leq\left(|R|_{H^{N-1}}+\mu C\left(\mu_{0}, \frac{1}{h_{\min }},|U|_{X_{\mu}^{N}},|(\tilde{\zeta}, \tilde{\mathbf{V}})|_{X_{\mu}^{N}},|R|_{H^{N-1}}\right)|\mathfrak{e}|_{X_{\mu}^{N-1}}\right)|\mathfrak{e}|_{X_{\mu}^{N-1}}
$$

Then the result follows from Gronwall's inequality.

We can now rigorously justify the Boussinesq-Coriolis equations. We recall that the operator $\overline{\mathbf{V}}[\varepsilon \zeta]\left(\mathbf{U}_{\|}^{\mu, \gamma}, \boldsymbol{\omega}\right)$ is defined in (9).

Theorem 2.9. Let $\mathbf{N} \geq 12$ and $(\mu, \varepsilon, \gamma, \mathrm{Ro}) \in \mathcal{A}_{\text {Boussi }}$. We assume that we are under the assumptions of Theorem 1.3. We define the following quantities

$$
\overline{\mathbf{V}}_{0}=\overline{\mathbf{V}}\left[\varepsilon \zeta_{0}\right]\left(\left(\mathbf{U}_{\|}^{\mu, \gamma}\right)_{0}, \boldsymbol{\omega}_{0}\right), \overline{\mathbf{V}}=\overline{\mathbf{V}}[\varepsilon \zeta]\left(\mathbf{U}_{\|}^{\mu, \gamma}, \boldsymbol{\omega}\right)
$$


Then, there exists a time $T>0$ such that

(i) $T$ has the form

$$
T=\frac{T_{0}}{\max \left(\mu, \frac{\varepsilon}{R o}\right)}, \text { and } \frac{1}{T_{0}}=c^{1}
$$

(ii) There exists a unique classical solution $\left(\zeta_{B}, \overline{\mathbf{V}}_{B}\right)$ of (3) with the initial data $\left(\zeta_{0}, \overline{\mathbf{V}}_{0}\right)$ on $[0, T]$.

(iii) There exists a unique classical solution $\left(\zeta, \mathbf{U}_{\|}^{\mu, \gamma}, \boldsymbol{\omega}\right)$ of System (7) with initial data $\left(\zeta_{0},\left(\mathbf{U}_{/ /}^{\mu, \gamma}\right)_{0}, \boldsymbol{\omega}_{0}\right)$ on $[0, T]$.

(iv) The following error estimate holds, for $0 \leq t \leq T$,

$$
\left|(\zeta, \overline{\mathbf{V}})-\left(\zeta_{B}, \overline{\mathbf{V}}_{B}\right)\right|_{L^{\infty}\left([0, t] \times \mathbb{R}^{2}\right)} \leq \mu^{2} t c^{2},
$$

with $c^{j}=C\left(A, \mu_{0}, \frac{1}{h_{\min }}, \frac{1}{\mathfrak{a}_{\min }}\right)$.

Therefore, in the Boussinesq regime $\mathcal{A}_{\text {Boussi }}$ a solution of the water waves system (7) remains close to a solution of the Boussinesq-Coriolis equations (3) over a time $\mathcal{O}\left(\frac{1}{\sqrt{\mu}}\right)$ with an accuracy of order $\mathcal{O}\left(\mu^{\frac{3}{2}}\right)$.

Remark 2.10. Notice that if one considers a solution of a system and wants to show that this solution remains close to a solution of the waves equations over times $\mathcal{O}\left(\frac{1}{\sqrt{\mu}}\right)$ with an accuracy of order $\mathcal{O}\left(\mu^{\frac{3}{2}}\right)$, it is sufficient to compare this solution with a solution of the Boussinesq-Coriolis equations (31). We use this strategy in the following.

\section{The KP approximation}

In this section, we consider the KP (Kadomtsev-Petviashvili) approximation under a weak Coriolis forcing. We assume that $\varepsilon=\mu$ (long waves) and $\gamma=\sqrt{\mu}$ (weakly transverse effects). We consider two different regimes. First, if $\frac{\varepsilon}{R_{0}}=\sqrt{\mu}$ (weak rotation), we derive the rotation-modified KP equation (12). Then, if $\frac{\varepsilon}{\text { Ro }_{0}}=\mu$ (very weak rotation), we derive the KP equation (19). We refer to [10] for more physical explanations about these two models (see also [11, 7, 9]).

\subsection{Weak rotation, the rotation-modified KP equation}

In the irrotational setting, the KP equation provides a good approximation of the water waves equation under the assumption that $\varepsilon$ and $\mu$ have the same order and that $\gamma$ and $\sqrt{\mu}$ have the same order (see [13] or Part 7.2 in [12]). When a Coriolis forcing is taken into account, Grimshaw and Melville ([10]) derived an equation for long waves, which is an adaptation of the KP equation 


$$
\partial_{\xi}\left(\partial_{\tau} k+\frac{3}{2} k \partial_{\xi} k+\frac{1}{6} \partial_{\xi}^{3} k\right)+\frac{1}{2} \partial_{y y} k=\frac{1}{2} k .
$$

This equation is called the rotation-modified KP equation or Grimshaw-Melville equation in the physics literature. Notice that this equation was originally derived for internal water waves $([10,7])$. In this part, we fully justify this equation. Inspired by [10, 7], we consider the asymptotic regime

$$
\mathcal{A}_{\mathrm{RKP}}=\left\{(\mu, \varepsilon, \gamma, \mathrm{Ro}), 0 \leq \mu \leq \mu_{0}, \varepsilon=\mu, \gamma=\sqrt{\mu}, \frac{\varepsilon}{\mathrm{Ro}}=\sqrt{\mu}\right\} .
$$

Then, the Boussinesq-Coriolis equations become $(\gamma=\sqrt{\mu})$

$$
\left\{\begin{array}{l}
\partial_{t} \zeta+\nabla^{\gamma} \cdot([1+\mu \zeta] \overline{\mathbf{V}})=0 \\
\left(1-\frac{\mu}{3} \nabla^{\gamma} \nabla^{\gamma} \cdot\right) \partial_{t} \overline{\mathbf{V}}+\nabla^{\gamma} \zeta+\mu \overline{\mathbf{V}} \cdot \nabla^{\gamma} \overline{\mathbf{V}}+\sqrt{\mu} \overline{\mathbf{V}}^{\perp}=0 .
\end{array}\right.
$$

In the following, we denote $\mathbf{V}=(u, v)^{t}$. Our strategy is similar to the one used in [16] to fully justify the Ostrovsky equation. We consider an expansion of $(\zeta, \mathbf{V})$ with respect to $\mu$. Inspired by [13] or Part 7.2 in [12], we seek an approximate solution $\left(\zeta_{\text {app }}, u_{\text {app }}, v_{\text {app }}\right)$ of (13) in the form

$$
\begin{aligned}
& \zeta_{a p p}(t, x, y)=k(x-t, y, \mu t)+\mu \zeta_{(1)}(t, x, y, \mu t), \\
& u_{a p p}(t, x, y)=k(x-t, y, \mu t)+\mu u_{(1)}(t, x, y, \mu t), \\
& v_{\text {app }}(t, x, y)=\sqrt{\mu} v_{(1 / 2)}(t, x, y, \mu t)
\end{aligned}
$$

where $k=k(\xi, \tau)$ is a traveling water wave modulated by a slow time variable and the others terms are correctors. In the following, we denote by $\tau$ the variable associated to the slow time variable $\mu t$. Plugging the ansatz into Sytem (13), we obtain

$$
\left\{\begin{array}{l}
\partial_{t} \zeta_{a p p}+\nabla^{\gamma} \cdot\left(\left[1+\mu \zeta_{a p p}\right] \overline{\mathbf{V}}_{a p p}\right)=\mu R_{(1)}^{1}+\mu^{2} R_{1} \\
\left(1-\frac{\mu}{3} \nabla^{\gamma} \nabla^{\gamma} \cdot\right) \partial_{t} \overline{\mathbf{V}}_{a p p}+\nabla^{\gamma} \zeta_{a p p}+\mu \overline{\mathbf{V}}_{a p p} \cdot \nabla^{\gamma} \overline{\mathbf{V}}_{a p p}+\sqrt{\mu} \overline{\mathbf{V}}_{a p p}^{\perp}=\sqrt{\mu} R_{(1 / 2)}^{2}+\mu R_{(1)}^{2}+\mu^{\frac{3}{2}} R_{2}
\end{array}\right.
$$

where

$$
\begin{aligned}
& R_{(1)}^{1}=\partial_{t} \zeta_{(1)}+\partial_{x} u_{(1)}+\partial_{\tau} k+2 k \partial_{\xi} k+\partial_{y} v_{(1 / 2)}, \\
& R_{(1 / 2)}^{2}=\left(\begin{array}{c}
0 \\
\partial_{t} v_{(1 / 2)}+\partial_{y} k+k
\end{array}\right) \text { and } R_{(1)}^{2}=\left(\begin{array}{c}
\partial_{t} u_{(1)}+\partial_{x} \zeta_{(1)}+\partial_{\tau} k+\frac{1}{3} \partial_{\xi}^{3} k+k \partial_{\xi} k-v_{(1 / 2)} \\
0
\end{array}\right),
\end{aligned}
$$

and

$$
\begin{aligned}
& R_{1}=\partial_{\tau} \zeta_{(1)}+\partial_{x}\left(k u_{(1)}+k \zeta_{(1)}+\mu \zeta_{(1)} u_{(1)}\right)+\partial_{y}\left(\left(k+\mu \zeta_{(1)}\right) v_{(1 / 2)}\right) \\
& R_{2}=\left(\sqrt{\mu} R_{2,1}, R_{2,2}\right)
\end{aligned}
$$

with 


$$
\begin{gathered}
R_{2,1}=\partial_{\tau} u_{(1)}-\frac{1}{3} \partial_{\xi}^{2} \partial_{\tau} k-\frac{1}{3} \partial_{x}^{2} \partial_{t} u_{(1)}-\mu \frac{1}{3} \partial_{x}^{2} \partial_{\tau} u_{(1)}+\partial_{x}\left(k u_{(1)}\right)+\mu u_{(1)} \partial_{x} u_{(1)} \\
-\frac{1}{3} \partial_{x y t}^{3} v_{(1 / 2)}-\frac{\mu}{3} \partial_{x y \tau}^{3} v_{(1 / 2)}+v_{(1 / 2)} \partial_{y}\left(k+\mu u_{(1)}\right) \\
R_{2,2}=\partial_{\tau} v_{(1 / 2)}+\partial_{y} \zeta_{(1)}+k \partial_{x} v_{(1 / 2)}+u_{(1)}+\frac{1}{3} \partial_{y} \partial_{\xi}^{2} k+\mu u_{(1)} \partial_{x} v_{(1 / 2)}+\mu v_{(1 / 2)} \partial_{y} v_{(1 / 2)} \\
-\frac{\mu}{3}\left(\partial_{y x \tau}^{3} k+\partial_{y x t}^{3} u_{(1)}+\partial_{y}^{2} \partial_{t} v_{(1 / 2)}+\mu \partial_{y x \tau} u_{(1)}+\mu \partial_{y}^{2} \partial_{\tau} v_{(1 / 2)}\right)
\end{gathered}
$$

Then, the strategy is to choose $\left(k, v_{(1 / 2)}\right)$ such that, for all $(x, y) \in \mathbb{R}^{2}, t \in\left[0, \frac{T}{\mu}\right]$ and $\tau \in[0, T]$,

$$
R_{(1)}^{1}(t, x, y, \tau)=0 \text { and } R_{(1 / 2)}^{2}(t, x, y, \tau)=R_{(1)}^{2}(t, x, y, \tau)=0 .
$$

Remark 3.1. As noticed in Part 7.2.2 in [12], we should a priori add $\sqrt{\mu} \zeta_{(1 / 2)}(t, x, y, \mu t)$, $\sqrt{\mu} u_{(1 / 2)}(t, x, y, \mu t), v_{(0)}(t, x, y, \mu t)$, and $\mu v_{(1)}(t, x, y, \mu t)$ to the ansatz (14) for $\zeta_{a p p}, u_{\text {app }}$ and $v_{\text {app }}$ respectively. But, it leads to $\zeta_{(1 / 2)}=u_{(1 / 2)}=v_{(0)}=v_{(1)}=0$ if these quantities are initially zero.

We focus first on the condition $R_{(1 / 2)}^{2}(t, x, y, \tau)=0$. Assuming that $v_{(1 / 2)}$ and $k$ vanish at $x=\infty$, this condition is equivalent to the equation

$$
\partial_{t} \partial_{x} v_{(1 / 2)}(t, x, y, \tau)+\partial_{\xi} k(x-t, y, \tau)+\partial_{\xi y}^{2} k(x-t, y, \tau)=0 .
$$

Then, using the fact that $\partial_{t}(k(x-t, y, \tau))=-\partial_{\xi} k(x-t, y, \tau)$, we can integrate with respect to $t$ and we get

$\partial_{x} v_{(1 / 2)}(t, x, y, \tau)=\partial_{x} v_{(1 / 2)}^{0}(x, y)+k(x-t, y, \tau)+\partial_{y} k(x-t, y, \tau)-k^{0}(x, y)-\partial_{y} k^{0}(x-t, y, \tau)$, where $k^{0}$ and $v_{(1 / 2)}^{0}$ are the initial data of $k$ and $v_{(1 / 2)}$ respectively. Then, assuming that $k(\cdot, \tau) \in \partial_{x} H^{N}\left(\mathbb{R}^{2}\right)$ for all $\tau \in[0, T]$ (see (4) ), we obtain

$$
\begin{aligned}
v_{(1 / 2)}(t, x, y, \tau)= & v_{(1 / 2)}^{0}(x, y)+\partial_{x}^{-1} k(x-t, y, \tau)+\partial_{x}^{-1} \partial_{y} k(x-t, y, \tau) \\
& -\partial_{x}^{-1} k^{0}(x, y)-\partial_{x}^{-1} \partial_{y} k^{0}(x-t, y, \tau),
\end{aligned}
$$

Secondly, we study the conditions $R_{(1)}^{1}=R_{(1)}^{2}=0$. Denoting $w_{ \pm}=\zeta_{(1)} \pm u_{(1)}$, we obtain

$$
\begin{aligned}
& \left(\partial_{t}+\partial_{x}\right) w_{+}+\left(2 \partial_{\tau} k+3 k \partial_{\xi} k+\frac{1}{3} \partial_{\xi}^{3} k+\partial_{\xi}^{-1} \partial_{y}^{2} k-\partial_{\xi}^{-1} k\right)(x-t, \tau)+F_{0}^{1}=0 \\
& \left(\partial_{t}-\partial_{x}\right) w_{-}+\left(k \partial_{\xi} k-\frac{1}{3} \partial_{\xi}^{3} k+\partial_{\xi}^{-1} \partial_{y}^{2} k+2 \partial_{\xi}^{-1} \partial_{y} k+\partial_{\xi}^{-1} k\right)(x-t, \tau)+F_{0}^{2}=0
\end{aligned}
$$

where 


$$
\begin{aligned}
& F_{0}^{1}=\partial_{y} v_{(1 / 2)}^{0}-v_{(1 / 2)}^{0}+\partial_{\xi}^{-1} k^{0}-\partial_{\xi}^{-1} \partial_{y}^{2} k^{0}, \\
& F_{0}^{2}=\partial_{y} v_{(1 / 2)}^{0}+v_{(1 / 2)}^{0}-\partial_{\xi}^{-1} k^{0}-\partial_{\xi}^{-1} \partial_{y}^{2} k^{0}-2 \partial_{\xi}^{-1} \partial_{y} k^{0} .
\end{aligned}
$$

The following Lemma (see Lemma 7.20 in [12] or Lemma 2 in [13]) gives us a Condition to control $\zeta_{(1)}$ and $u_{(1)}$.

Lemma 3.2. Let $c_{1} \neq c_{2}$. Let $k_{1}, k_{2} \in H^{2}\left(\mathbb{R}^{2}\right)$ with $k_{2}=\partial_{x} K_{2}$ and $K_{2} \in H^{3}\left(\mathbb{R}^{2}\right)$. We consider the unique solution $k$ of

$$
\left\{\begin{array}{l}
\left(\partial_{t}+c_{1} \partial_{x}\right) k=k_{1}\left(x-c_{1} t, y\right)+k_{2}\left(x-c_{2} t, y\right), \\
k_{\mid t=0}=0 .
\end{array}\right.
$$

Then, $\lim _{t \rightarrow \infty}\left|\frac{1}{t} k(t, \cdot)\right|_{H^{2}}=0$ if and only if $k_{1} \equiv 0$ and in that case

$$
|k(t, \cdot)|_{H^{2}} \leq C \frac{t}{1+t}\left|K_{2}\right|_{H^{3}} .
$$

Hence, since we want to avoid a linear growth of the solution of (17), we must impose

$$
\partial_{\tau} k+\frac{3}{2} k \partial_{\xi} k+\frac{1}{6} \partial_{\xi}^{3} k+\frac{1}{2} \partial_{\xi}^{-1} \partial_{y}^{2} k-\frac{1}{2} \partial_{\xi}^{-1} k=0
$$

which is the the rotation-modified KP equation defined in (12). In the following, we provide a local existence result for this equation. This proposition generalizes Theorem 1.1 in [5].

Proposition 3.3. Let $N \geq 4$ and $k_{0} \in \partial_{x} H^{N}\left(\mathbb{R}^{2}\right)$. Then, there exists a time $T>0$ and a unique solution $k \in \mathcal{C}\left([0, T] ; \partial_{x} H^{N}\left(\mathbb{R}^{2}\right)\right)$ to the rotation-modified KP equation (18) and one has

$$
\left|\partial_{\xi}^{-1} k(t, \cdot)\right|_{H^{N}} \leq C\left(T,\left|\partial_{\xi}^{-1} k_{0}\right|_{H^{N}}\right) .
$$

Furthermore, if $k_{0}, \partial_{y}^{2} k_{0} \in \partial_{x}^{2} H^{N-2}\left(\mathbb{R}^{2}\right)$, then $k \in \mathcal{C}\left([0, T] ; \partial_{x}^{2} H^{N-2}\left(\mathbb{R}^{2}\right)\right)$ and one has

$$
\left|\partial_{\xi}^{-2} k(t, \cdot)\right|_{H^{N-2}} \leq C\left(T,\left|\partial_{\xi}^{-2} k_{0}\right|_{H^{N-2}},\left|\partial_{\xi}^{-2} \partial_{y}^{2} k_{0}\right|_{H^{N-2}},\left|\partial_{\xi} k_{0}\right|_{H^{N}}\right) .
$$

Finally, if $N \geq 6$ and $k_{0}, \partial_{y}^{2} k_{0} \in \partial_{x}^{2} H^{N-4}\left(\mathbb{R}^{2}\right)$, then $\partial_{y}^{2} k \in \mathcal{C}\left([0, T] ; \partial_{x}^{2} H^{N-4}\left(\mathbb{R}^{2}\right)\right)$ and one has

$$
\left|\partial_{\xi}^{-2} k(t, \cdot)\right|_{H^{N-4}} \leq C\left(T,\left|\partial_{\xi}^{-2} k_{0}\right|_{H^{N-4}},\left|\partial_{\xi}^{-2} \partial_{y}^{2} k_{0}\right|_{H^{N-4}},\left|\partial_{\xi}^{-1} k_{0}\right|_{H^{N}}\right)
$$


Proof. The first point follows from Theorem 1.1 in [5]. We only have to prove the second and the third points. This proof is similar to the proof of Lemma 7.22 in [12] for the $\mathrm{KP}$ equation and the proof of Proposition 3.8 in [16] for the Ostrovsky equation. In the following, we denote by $S(t)$ the semi-group of the linearized rotation-modified KP equation

$$
\partial_{\tau} k+\frac{1}{6} \partial_{\xi}^{3} k+\frac{1}{2} \partial_{\xi}^{-1} \partial_{y}^{2} k-\frac{1}{2} \partial_{\xi}^{-1} k=0 .
$$

One can check that this semi-group acts unitary on $H^{N}\left(\mathbb{R}^{2}\right)$. We also define $\tilde{k}=\partial_{\tau} k$. We can check that

$$
\partial_{\tau} \tilde{k}+\frac{1}{6} \partial_{\xi}^{3} \tilde{k}+\frac{1}{2} \partial_{\xi}^{-1} \partial_{y}^{2} \tilde{k}-\frac{1}{2} \partial_{\xi}^{-1} \tilde{k}+\frac{3}{2} \partial_{\xi}(\tilde{k} k)=0
$$

Using the Duhamel's formula we obtain

$$
\partial_{\xi}^{-1} \tilde{k}(\tau)=S(t) \partial_{\xi}^{-1} \tilde{k}_{0}-\frac{3}{2} \int_{0}^{\tau} S(t-s)(k(s) \tilde{k}(s)) d s .
$$

We can see by product estimates that $\partial_{\xi}^{-1} \tilde{k}_{0}, k(s) \tilde{k}(s) \in H^{N-4}\left(\mathbb{R}^{2}\right)$ and then that $\tilde{k} \in \mathcal{C}\left([0, T] ; \partial_{x} H^{N-3}\left(\mathbb{R}^{2}\right)\right)$. Then, we consider the following equality

$$
\frac{1}{2}\left(1-\partial_{y}^{2}\right) \partial_{\xi}^{-2} k=\partial_{\xi}^{-1} \tilde{k}+\frac{3}{4} k^{2}+\frac{1}{6} \partial_{\xi}^{2} k
$$

For the second point, we get that $\left(1-\partial_{\xi}^{2}-\partial_{y}^{2}\right) \partial_{\xi}^{-2} k \in H^{N-4}\left(\mathbb{R}^{2}\right)$ and the result follows easily. For the third point, we obtain from the second point that $\partial_{y}^{2} \partial_{\xi}^{-2} k \in H^{N-4}\left(\mathbb{R}^{2}\right)$.

We can now rigorously justify the rotation-modified KP equation. The following theorem is the main theorem of this part.

Theorem 3.4. Let $k^{0} \in \partial_{x}^{2} H^{12}\left(\mathbb{R}^{2}\right)$ such that $1+\varepsilon k^{0} \geq h_{\min }>0$ and $v^{0} \in \partial_{x} H^{8}\left(\mathbb{R}^{2}\right)$. Suppose that $(\mu, \varepsilon, \gamma, \mathrm{Ro}) \in \mathcal{A}_{\mathrm{RKP}}$. Then, there exists a time $T_{0}>0$, such that we have

(i) a unique classical solution $\left(\zeta_{B}, u_{B}, v_{B}\right)$ of (13) with initial data $\left(k^{0}, k^{0}, \sqrt{\mu} v^{0}\right)$ on $\left[0, \frac{T_{0}}{\mu}\right]$.

(ii) a unique classical solution $k$ of (18) with initial data $k^{0}$ on $\left[0, T_{0}\right]$.

(iii) If we define $\left(\zeta_{R K P}, u_{R K P}\right)(t, x, y)=(k(x-t, y, \mu t), k(x-t, y, \mu t))$ we have the following error estimate for all $0 \leq t \leq \frac{T_{0}}{\mu}$,

$$
\left|\left(\zeta_{B}, u_{B}\right)-\left(\zeta_{R K P}, u_{R K P}\right)\right|_{L^{\infty}\left([0, t] \times \mathbb{R}^{2}\right)} \leq C \frac{\mu t}{1+t}(1+\sqrt{\mu} t)
$$

where $C=C\left(\frac{1}{h_{\min }}, \mu_{0},\left|\partial_{x}^{-2} k^{0}\right|_{H^{12}},\left|\partial_{x}^{-1} v^{0}\right|_{H^{8}}\right)$. 
Proof. In order to simplify the technicality of this proof, $C$ is a constant of the form

$$
C=C\left(\frac{1}{h_{\min }}, \mu_{0},\left|\partial_{x}^{-2} k^{0}\right|_{H^{12}},\left|\partial_{x}^{-1} v^{0}\right|_{H^{9}}\right)
$$

The first and second point follow from Proposition 2.7 and Proposition 3.3. Then, from System (17) and Lemma 3.2, we obtain

$$
\left|\zeta_{(1)}\right|_{H^{2}}+\left|u_{(1)}\right|_{H^{2}} \leq C \frac{t}{1+t}
$$

We also notice that we can control all the derivatives with respect to $x, y$ or $\tau$ of $u$ and $v$ be differentiating (17). Hence, we get a control for the remainders $R_{1}$ and $R_{2}$ and we obtain, for $0 \leq t \leq \frac{T}{\mu}$

$$
\left|R_{1}(t)\right|_{H^{3}}+\left|R_{2}(t)\right|_{H^{3}} \leq C .
$$

Then, using Proposition 2.8, on can have

$$
\left|\left(\zeta_{B}, u_{B}, v_{B}\right)-\left(\zeta_{a p p}, u_{a p p}, v_{a p p}\right)\right|_{L^{\infty}\left([0, t] \times \mathbb{R}^{2}\right)} \leq C \mu^{\frac{3}{2}} t .
$$

Finally, from the ansatz (14) and Lemma $\underline{3.2}$, we have

$$
\left|\left(\zeta_{\text {app }}, u_{\text {app }}\right)-\left(\zeta_{R K P}, v_{R K P}\right)\right|_{L^{\infty}\left([0, t] \times \mathbb{R}^{2}\right)} \leq \mu \frac{t}{1+t},
$$

and the result follows easily.

This theorem provides the first mathematical justification of the rotation-modified KP equation. Notice that the condition $k^{0} \in \partial_{x}^{2} H^{10}\left(\mathbb{R}^{2}\right)$ is quite restrictive. As noted in [12] Part 7.2.1 and in [16] for the Ostrovsky equation, using the strategy developed in [1], we can hope to weaken the assumption on $k^{0}$ into $k^{0} \in \partial_{x} H^{9}\left(\mathbb{R}^{2}\right)$.

\subsection{Very weak rotation, the KP equation}

In this part we study the situation of a very weak Coriolis forcing. We derive and fully justify the KP equation. We show that if $\frac{\varepsilon}{\text { Ro }}$ is small enough, we can derive the KP equation

$$
\partial_{\xi}\left(\partial_{\tau} k+\frac{3}{2} k \partial_{\xi} k+\frac{1}{6} \partial_{\xi}^{3} k\right)+\frac{1}{2} \partial_{y y} k=0 .
$$

Inspired by [10], we consider the following asymptotic regime

$$
\mathcal{A}_{\mathrm{KP}}=\left\{(\mu, \varepsilon, \gamma, \mathrm{Ro}), 0 \leq \mu \leq \mu_{0}, \varepsilon=\mu, \gamma=\sqrt{\mu}, \frac{\varepsilon}{\mathrm{Ro}}=\mu\right\} .
$$

The Boussinesq-Coriolis equations become $(\gamma=\sqrt{\mu})$ 


$$
\left\{\begin{array}{l}
\partial_{t} \zeta+\nabla^{\gamma} \cdot([1+\mu \zeta] \overline{\mathbf{V}})=0 \\
\left(1-\frac{\mu}{3} \nabla^{\gamma} \nabla^{\gamma} \cdot\right) \partial_{t} \overline{\mathbf{V}}+\nabla^{\gamma} \zeta+\mu \overline{\mathbf{V}} \cdot \nabla^{\gamma} \overline{\mathbf{V}}+\mu \overline{\mathbf{V}}^{\perp}=0 .
\end{array}\right.
$$

Proceeding as in the previous part, we denote $\mathbf{V}=(u, v)^{t}$ and we seek an approximate solution $\left(\zeta_{a p p}, u_{a p p}, v_{a p p}\right)$ of (20) in the form

$$
\begin{aligned}
& \zeta_{\text {app }}(t, x)=k(x-t, y, \mu t)+\mu \zeta_{(1)}(t, x, y, \mu t), \\
& u_{\text {app }}(t, x)=k(x-t, y, \mu t)+\mu u_{(1)}(t, x, y, \mu t), \\
& v_{\text {app }}(t, x)=\sqrt{\mu} v_{(1 / 2)}(t, x, y, \mu t)+\mu v_{(1)}(t, x, y, \mu t) .
\end{aligned}
$$

Then, we plug the ansatz into Sytem (20) and we get

$$
\left\{\begin{array}{l}
\partial_{t} \zeta_{a p p}+\nabla^{\gamma} \cdot\left(\left[1+\mu \zeta_{a p p}\right] \overline{\mathbf{V}}_{a p p}\right)=\mu R_{(1)}^{1}+\mu^{\frac{3}{2}} \partial_{y} v_{(1)}+\mu^{2} R_{1}, \\
\left(1-\frac{\mu}{3} \nabla^{\gamma} \nabla^{\gamma} \cdot\right) \partial_{t} \overline{\mathbf{V}}_{a p p}+\nabla^{\gamma} \zeta_{a p p}+\mu \overline{\mathbf{V}}_{a p p} \cdot \nabla^{\gamma} \overline{\mathbf{V}}_{a p p}+\mu \overline{\mathbf{V}}_{a p p}^{\perp}=\sqrt{\mu} R_{(1 / 2)}^{2}+\mu R_{(1)}^{2}+\mu^{\frac{3}{2}} R_{2}
\end{array}\right.
$$

where

$$
\begin{aligned}
& R_{(1)}^{1}=\partial_{t} \zeta_{(1)}+\partial_{x} u_{(1)}+\partial_{\tau} k+2 k \partial_{\xi} k+\partial_{y} v_{(1 / 2)}, \\
& R_{(1 / 2)}^{2}=\left(\begin{array}{c}
0 \\
\partial_{t} v_{(1 / 2)}+\partial_{y} k
\end{array}\right) \text { and } R_{(1)}^{2}=\left(\begin{array}{c}
\partial_{t} u_{(1)}+\partial_{x} \zeta_{(1)}+\partial_{\tau} k+\frac{1}{3} \partial_{\xi}^{3} k+k \partial_{\xi} k \\
\partial_{t} v_{(1)}+k
\end{array}\right),
\end{aligned}
$$

and

$$
\begin{aligned}
& R_{1}=\partial_{\tau} \zeta_{(1)}+\partial_{x}\left(k u_{(1)}+k \zeta_{(1)}+\mu \zeta_{(1)} u_{(1)}\right)+\partial_{y}\left(\left(k+\mu \zeta_{(1)}\right)\left(v_{(1 / 2)}+\mu v_{(1)}\right)\right), \\
& R_{2}=\left(-\left(v_{(1 / 2)}+\sqrt{\mu} v_{(1)}\right)+\sqrt{\mu} \tilde{R}_{2,1}, R_{2,2}\right)
\end{aligned}
$$

with

$$
\begin{aligned}
\tilde{R}_{2,1}= & \partial_{\tau} u_{(1)}-\frac{1}{3} \partial_{\xi}^{2} \partial_{\tau} k-\frac{1}{3} \partial_{x}^{2} \partial_{t} u_{(1)}-\mu \frac{1}{3} \partial_{x}^{2} \partial_{\tau} u_{(1)}+\partial_{x}\left(k u_{(1)}\right)+\mu u_{(1)} \partial_{x} u_{(1)} \\
& -\frac{1}{3} \partial_{x y t}^{3}\left(v_{(1 / 2)}+\sqrt{\mu} v_{(1)}\right)-\frac{\mu}{3} \partial_{x y \tau}^{3}\left(v_{(1 / 2)}+\sqrt{\mu} v_{(1)}\right)+\left(v_{(1 / 2)}+\sqrt{\mu} v_{(1)}\right) \partial_{y}\left(k+\mu u_{(1)}\right), \\
R_{2,2}= & \partial_{\tau} v_{(1 / 2)}+\partial_{y} \zeta_{(1)}+k \partial_{x}\left(v_{(1 / 2)}+\sqrt{\mu} v_{(1)}\right)+u_{(1)}+\frac{1}{3} \partial_{y} \partial_{\xi}^{2} k+\mu u_{(1)} \partial_{x}\left(v_{(1 / 2)}+\sqrt{\mu} v_{(1)}\right) \\
& -\frac{\mu}{3}\left(\partial_{y x \tau}^{3} k+\partial_{y x t}^{3} u_{(1)}+\partial_{y}^{2} \partial_{t}\left(v_{(1 / 2)}+\sqrt{\mu} v_{(1)}\right)+\mu \partial_{y x \tau} u_{(1)}+\mu \partial_{y}^{2} \partial_{\tau}\left(v_{(1 / 2)}+\sqrt{\mu} v_{(1)}\right)\right) \\
& +\mu\left(v_{(1 / 2)}+\sqrt{\mu} v_{(1)}\right) \partial_{y}\left(v_{(1 / 2)}+\sqrt{\mu} v_{(1)}\right) .
\end{aligned}
$$

Then, we choose $\left(k, v_{(1 / 2)}, v_{(1)}\right)$ such that, for all $(x, y) \in \mathbb{R}^{2}, t \in\left[0, \frac{T}{\mu}\right]$ and $\tau \in[0, T]$,

$$
R_{(1)}^{1}(t, x, y, \tau)=0 \text { and } R_{(1 / 2)}^{2}(t, x, y, \tau)=R_{(1)}^{2}(t, x, y, \tau)=0 .
$$

First, we obtain that 


$$
\begin{aligned}
& v_{(1 / 2)}=\partial_{x}^{-1} \partial_{y} k+v_{(1 / 2)}^{0}-\partial_{x}^{-1} \partial_{y} k^{0}, \\
& v_{(1)}=\partial_{x}^{-1} k+v_{(1)}^{0}-\partial_{x}^{-1} k^{0} .
\end{aligned}
$$

Then, denoting $w_{ \pm}=\zeta_{(1)} \pm u_{(1)}$, we get

$$
\begin{aligned}
& \left(\partial_{t}+\partial_{x}\right) w_{+}+\left(2 \partial_{\tau} k+3 k \partial_{\xi} k+\frac{1}{3} \partial_{\xi}^{3} k+\partial_{\xi}^{-1} \partial_{y}^{2} k\right)(x-t, \tau)+F_{0}=0, \\
& \left(\partial_{t}-\partial_{x}\right) w_{-}+\left(k \partial_{\xi} k-\frac{1}{3} \partial_{\xi}^{3} k+\partial_{\xi}^{-1} \partial_{y}^{2} k\right)(x-t, \tau)+F_{0}=0
\end{aligned}
$$

where

$$
F_{0}=\partial_{y} v_{(1 / 2)}^{0}-\partial_{\xi}^{-1} \partial_{y}^{2} k^{0} .
$$

Therefore, in order to avoid a linear growth (see Lemma 3.2), $k$ must satisfies the KP equation (19). The following Lemma is a local wellposedness result for the KP equation (see Lemma 7.22 in [12] or [19, 2, 21]).

Proposition 3.5. Let $N \geq 5$ and $k_{0} \in \partial_{x} H^{N}\left(\mathbb{R}^{2}\right)$. Then, there exists a time $T>0$ and a unique solution $k \in \mathcal{C}\left([0, T] ; \partial_{x} H^{N}\left(\mathbb{R}^{2}\right)\right)$ to the KP equation (19) and one has

$$
\left|\partial_{\xi}^{-1} k(t, \cdot)\right|_{H^{N}} \leq C\left(T,\left|\partial_{\xi}^{-1} k_{0}\right|_{H^{N}}\right) \text {. }
$$

Furthermore, if $N \geq 6$ and $\partial_{y}^{2} k_{0} \in \partial_{x}^{2} H^{N-4}\left(\mathbb{R}^{2}\right)$, then $\partial_{y}^{2} k \in \mathcal{C}\left([0, T] ; \partial_{x}^{2} H^{N-4}\left(\mathbb{R}^{2}\right)\right)$ and one has

$$
\left|\partial_{y}^{2} k_{0} \partial_{\xi}^{-2} k(t, \cdot)\right|_{H^{N-4}} \leq C\left(T,\left|\partial_{\xi}^{-2} \partial_{y}^{2} k_{0}\right|_{H^{N-4}},\left|\partial_{\xi}^{-1} k_{0}\right|_{H^{N}}\right)
$$

We can now establish a rigorous justification of the KP equation.

Theorem 3.6. Let $k^{0} \in \partial_{x}^{2} H^{12}\left(\mathbb{R}^{2}\right)$ such that $1+\varepsilon k^{0} \geq h_{\min }>0$ and $v_{(1 / 2)}^{0} \in \partial_{x} H^{8}\left(\mathbb{R}^{2}\right)$, $v_{(1)}^{0} \in H^{7}\left(\mathbb{R}^{2}\right)$. Suppose that $(\mu, \varepsilon, \gamma, \mathrm{Ro}) \in \mathcal{A}_{\mathrm{KP}}$. Denote $v^{0}=\sqrt{\mu} v_{(1 / 2)}^{0}+\mu v_{(1)}^{0}$. Then, there exists a time $T_{0}>0$, such that we have

(i) a unique classical solution $\left(\zeta_{B}, u_{B}, v_{B}\right)$ of (13) with initial data $\left(k^{0}, k^{0}, v^{0}\right)$ on $\left[0, \frac{T_{0}}{\mu}\right]$.

(ii) a unique classical solution $k$ of (19) with initial data $k^{0}$ on $\left[0, T_{0}\right]$.

(iii) If we define $\left(\zeta_{K P}, u_{K P}\right)(t, x)=(k(x-t, y, \mu t), k(x-t, y, \mu t))$ we have the following error estimate for all $0 \leq t \leq \frac{T_{0}}{\mu}$,

$$
\left|\left(\zeta_{B}, u_{B}\right)-\left(\zeta_{K P}, u_{K P}\right)\right|_{L^{\infty}\left([0, t] \times \mathbb{R}^{2}\right)} \leq C \frac{\mu t}{1+t}(1+\sqrt{\mu} t)
$$

where $C=C\left(\frac{1}{h_{\min }}, \mu_{0},\left|\partial_{x}^{-2} k^{0}\right|_{H^{12}},\left|\partial_{x}^{-1} v_{(1 / 2)}^{0}\right|_{H^{8}},\left|v_{(1)}^{0}\right|_{H^{7}}\right)$. 
Proof. The proof is very similar to the proof of Theorem 3.4 .

Remark 3.7. Contrary to the justification of the KP equation in the irrotational setting (see Part 7.2 in [12] or [13]), the transverse part of the horizontal velocity $v$ must contain an order $\mathcal{O}(\mu)$ contribution. Notice that if one considers a weaker Coriolis forcing, for instance $\frac{\varepsilon}{R o}=\mu^{\frac{3}{2}}$, this assumption is no more necessary.

\section{Which equation for which asymptotic regime?}

\subsection{The Ostrovsky and KdV equations}

In Section 3, we derived two asymptotic models in the long wave regime $(\varepsilon=\mu)$. First, if $\gamma=\sqrt{\mu}$ and $\frac{\varepsilon}{\mathrm{Ro}_{0}}=\sqrt{\mu}$, we derived the rotation-modified KP equation

$$
\partial_{\xi}\left(\partial_{\tau} k+\frac{3}{2} k \partial_{\xi} k+\frac{1}{6} \partial_{\xi}^{3} k\right)+\frac{1}{2} \partial_{y y} k=\frac{1}{2} k .
$$

Then, if $\gamma=\sqrt{\mu}$ and $\frac{\varepsilon}{\text { Ro }}=\mu$, we obtained the KP equation

$$
\partial_{\xi}\left(\partial_{\tau} k+\frac{3}{2} k \partial_{\xi} k+\frac{1}{6} \partial_{\xi}^{3} k\right)+\frac{1}{2} \partial_{y y} k=0 .
$$

In [16], we performed a similar derivation in the long wave regime under the assumption that $\gamma=\mathcal{O}\left(\mu^{2}\right)$. When $\frac{\varepsilon}{R_{0}}=\sqrt{\mu}$, we derived the Ostrovsky equation

$$
\partial_{\xi}\left(\partial_{\tau} k+\frac{3}{2} k \partial_{\xi} k+\frac{1}{6} \partial_{\xi}^{3} k\right)=\frac{1}{2} k
$$

and when $\frac{\varepsilon}{\mathrm{Ro}}=\mu$, we derived the $\mathrm{KdV}$ equation

$$
\partial_{\tau} k+\frac{3}{2} k \partial_{\xi} k+\frac{1}{6} \partial_{\xi}^{3} k=0
$$

We would like to emphasize that we can weaken the assumption $\gamma=\mathcal{O}\left(\mu^{2}\right)$ into $\gamma=\mu$. In the following, we show this fact on the Ostrovsky equation. We consider the asymptotic regime

$$
\mathcal{A}_{\text {ostrov }}=\left\{(\mu, \varepsilon, \gamma, \text { Ro }), 0 \leq \mu \leq \mu_{0}, \varepsilon=\mu, \gamma=\mu, \frac{\varepsilon}{\operatorname{Ro}}=\sqrt{\mu}\right\} .
$$

Then we seek an approximate solution $\left(\zeta_{a p p}, u_{a p p}, v_{a p p}\right)$ of the Boussinesq-Coriolis equations in the form

$$
\begin{aligned}
& \zeta_{a p p}(t, x, y)=k(x-t, y, \mu t)+\mu \zeta_{(1)}(t, x, y, \mu t), \\
& u_{a p p}(t, x, y)=k(x-t, y, \mu t)+\mu u_{(1)}(t, x, y, \mu t), \\
& v_{a p p}(t, x, y)=\sqrt{\mu} v_{(1 / 2)}(t, x, y, \mu t)+\mu v_{(1)}(t, x, y, \mu t)
\end{aligned}
$$

Plugging the ansatz into the Boussinesq-Coriolis equations, we obtain 


$$
\left\{\begin{array}{l}
\partial_{t} \zeta_{a p p}+\nabla^{\gamma} \cdot\left(\left[1+\mu \zeta_{a p p}\right] \overline{\mathbf{V}}_{a p p}\right)=\mu R_{(1)}^{1}+\mu^{\frac{3}{2}} R_{1}, \\
\left(1-\frac{\mu}{3} \nabla^{\gamma} \nabla^{\gamma} \cdot\right) \partial_{t} \overline{\mathbf{V}}_{a p p}+\nabla^{\gamma} \zeta_{a p p}+\mu \overline{\mathbf{V}}_{a p p} \cdot \nabla^{\gamma} \overline{\mathbf{V}}_{a p p}+\sqrt{\mu} \overline{\mathbf{V}}_{a p p}^{\perp}=\sqrt{\mu} R_{(1 / 2)}^{2}+\mu R_{(1)}^{2}+\mu^{\frac{3}{2}} R_{2}
\end{array}\right.
$$

where

$$
\begin{aligned}
& R_{(1)}^{1}=\partial_{t} \zeta_{(1)}+\partial_{x} u_{(1)}+\partial_{\tau} k+2 k \partial_{\xi} k \\
& R_{(1 / 2)}^{2}=\left(\begin{array}{c}
0 \\
\partial_{t} v_{(1 / 2)}+k
\end{array}\right) \text { and } R_{(1)}^{2}=\left(\begin{array}{c}
\partial_{t} u_{(1)}+\partial_{x} \zeta_{(1)}+\partial_{\tau} k+\frac{1}{3} \partial_{\xi}^{3} k+k \partial_{\xi} k-v_{(1 / 2)} \\
\partial_{t} v_{(1)}+\partial_{y} k
\end{array}\right),
\end{aligned}
$$

and where $R_{1}, R_{2}$ are remainders similar to the ones found in Sections 3.1 and 3.2. Then, using the same strategy than before, we impose that $R_{(1)}^{1}=0$ and $R_{(1 / 2)}^{2}=R_{(1)}^{2}=0$. We obtain

$$
\begin{aligned}
& v_{(1 / 2)}=\partial_{\xi}^{-1} k+v_{(1 / 2)}^{0}-\partial_{\xi}^{-1} k^{0} \\
& v_{(1)}=\partial_{\xi}^{-1} \partial_{y} k+v_{(1)}^{0}-\partial_{\xi}^{-1} \partial_{y} k^{0}
\end{aligned}
$$

and, denoting $w_{ \pm}=\zeta_{(1)} \pm u_{(1)}$, we get

$$
\begin{aligned}
& \left(\partial_{t}+\partial_{x}\right) w_{+}+\left(2 \partial_{\tau} k+3 k \partial_{\xi} k+\frac{1}{3} \partial_{\xi}^{3} k-\partial_{\xi}^{-1} k\right)(x-t, \tau)-F_{0}=0, \\
& \left(\partial_{t}-\partial_{x}\right) w_{-}+\left(k \partial_{\xi} k-\frac{1}{3} \partial_{\xi}^{3} k+\partial_{\xi}^{-1} k\right)(x-t, \tau)+F_{0}=0,
\end{aligned}
$$

where $F_{0}=v_{(1 / 2)}^{0}-\partial_{\xi}^{-1} k^{0}$. In order to avoid a linear growth (see Lemma 3.2), $k$ must satisfies the Ostrovsky equation (22). Proceeding as in [16], we can generalize Theorem 3.9 in [16] to the asymptotic regime $\mathcal{A}_{\text {ostrov }}$. A solution of the Ostrovsky equation provides a $\mathcal{O}(\sqrt{\mu})$ approximation of the Boussinesq-Coriolis equations over a time $\mathcal{O}\left(\frac{1}{\mu}\right)$. We can proceed similarly for the $\mathrm{KdV}$ equation (23). Under the asymptotic regime

$$
\mathcal{A}_{\mathrm{KdV}}=\left\{(\mu, \varepsilon, \gamma, \mathrm{Ro}), 0 \leq \mu \leq \mu_{0}, \varepsilon=\mu, \gamma=\mu, \frac{\varepsilon}{\mathrm{Ro}}=\mu\right\} .
$$

and with the ansatz

$$
\begin{aligned}
& \zeta_{a p p}(t, x, y)=k(x-t, y, \mu t)+\mu \zeta_{(1)}(t, x, y, \mu t), \\
& u_{a p p}(t, x, y)=k(x-t, y, \mu t)+\mu u_{(1)}(t, x, y, \mu t), \\
& v_{a p p}(t, x, y)=\mu v_{(1)}(t, x, y, \mu t),
\end{aligned}
$$

we can generalize Theorem 3.12 in [16] to the asymptotic regime $\mathcal{A}_{\mathrm{KdV}}$. A solution of the $\mathrm{KdV}$ equation provides a $\mathcal{O}(\mu)$ approximation of the Boussinesq-Coriolis equations over a time $\mathcal{O}\left(\frac{1}{\mu}\right)$. 


\subsection{Conclusion}

We summarize Section 3 and Subsection 4.1 by the following table. Notice that all of these models provide a $\mathcal{O}(\sqrt{\mu})$ approximation (at least) in the long wave regime $(\varepsilon=\mu)$ of the Boussinesq-Coriolis equations over a time $\mathcal{O}\left(\frac{1}{\mu}\right)$.

\begin{tabular}{|c|c|c|}
\hline$\gamma \sqrt{\frac{\varepsilon}{R o}}$ & $\sqrt{\mu}$ & $\mu$ \\
\hline$\sqrt{\mu}$ & Rotation-modified KP equation & KP equation \\
\hline$\mu$ & Ostrovsky equation & KdV equation \\
\hline
\end{tabular}

\section{Acknowledgments}

The author would like to thank Jean-Claude Saut for the fruitful discussions about the KP approximation. 


\section{References}

[1] W. Ben Youssef and D. Lannes. The long wave limit for a general class of $2 \mathrm{~d}$ quasilinear hyperbolic problems. Communications in Partial Differential Equations, 27(5-6):979-1020, 2002.

[2] J. Bourgain. On the Cauchy problem for the Kadomtsev-Petviashvili equation. Geom. Funct. Anal., 3(4):315-341, 1993.

[3] A. Castro and D. Lannes. Fully nonlinear long-wave models in the presence of vorticity. Journal of Fluid Mechanics, 759:642-675, 112014.

[4] A. Castro and D. Lannes. Well-posedness and shallow-water stability for a new Hamiltonian formulation of the water waves equations with vorticity. Indiana Univ. Math. J., 64(4):1169-1270, 2015.

[5] R. M. Chen, V. M. Hur, and Y. Liu. Solitary waves of the rotation-modified Kadomtsev-Petviashvili equation. Nonlinearity, 21(12):2949-2979, 2008.

[6] W. Craig, C. Sulem, and P.-L. Sulem. Nonlinear modulation of gravity waves: a rigorous approach. Nonlinearity, 5(2):497-522, 1992.

[7] J. P. Germain and D. P. Renouard. On permanent nonlinear waves in a rotating fluid. Fluid Dynamics Research, 7(5-6):263, 1991.

[8] A.E. Gill. Atmosphere-Ocean Dynamics. International Geophysics. Elsevier Science, 1982.

[9] R. Grimshaw. Evolution equations for weakly nonlinear, long internal waves in a rotating fluid. Stud. Appl. Math., 73(1):1-33, 1985.

[10] R. Grimshaw and W. K. Melville. On the derivation of the modified Kadomtsev-Petviashvili equation. Stud. Appl. Math., 80(3):183-202, 1989.

[11] R.H.J. Grimshaw, L.A. Ostrovsky, V.I. Shrira, and Yu. A. Stepanyants. Long nonlinear surface and internal gravity waves in a rotating ocean. Surveys in Geophysics, 19(4):289-338, Jul 1998.

[12] D. Lannes. The water waves problem, volume 188 of Mathematical Surveys and Monographs. American Mathematical Society, Providence, RI, 2013. Mathematical analysis and asymptotics.

[13] D. Lannes and J. C. Saut. Weakly transverse Boussinesq systems and the Kadomtsev-Petviashvili approximation. Nonlinearity, 19(12):2853-2875, 2006.

[14] P.H. LeBlond and L.A. Mysak. Waves in the Ocean. Elsevier Oceanography Series. Elsevier Science, 1981.

[15] B. Melinand. Coriolis effect on water waves. ESAIM: M2AN, 51(5):1957-1985, 2017.

[16] B. Melinand. Long wave approximation for water waves under a coriolis forcing and the ostrovsky equation. Proc. R. Soc. Edinb., Sect. A, Math. ,To appear, 2017.

[17] J. Pedlosky. Geophysical Fluid Dynamics. Springer study edition. Springer New York, 1992.

[18] G. L. Richard and S. L. Gavrilyuk. A new model of roll waves: comparison with Brock's experiments. J. Fluid Mech., 698:374-405, 2012.

[19] J.C. Saut. Remarks on the generalized Kadomtsev-Petviashvili equations. Indiana Univ. Math. J., 42(3):1011-1026, 1993.

[20] V. M. Teshukov. Gas-dynamic analogy for vortex free-boundary flows. Journal of Applied Mechanics and Technical Physics, 48(3):303-309, 2007.

[21] S. Ukai. Local solutions of the Kadomtsev-Petviashvili equation. J. Fac. Sci. Univ. Tokyo Sect. IA Math., 36(2):193-209, 1989.

[22] V.E Zakharov. Stability of periodic waves of finite amplitude on the surface of a deep fluid. $J$. Applied Mech. Tech. Phys., 9:190-194, 1968. 